\title{
Acquisition geometry analysis in complex 3D media
}

\author{
E. J. van Veldhuizen ${ }^{1}$, G Blacquière ${ }^{2}$, and A. J. Berkhout ${ }^{2}$
}

\begin{abstract}
Increasingly, we must deal with complex subsurface structures in seismic exploration, often resulting in poor illumination and, therefore, poor image quality. Consequently, it is desirable to take into consideration the effects of wave propagation in the subsurface structure when designing an acquisition geometry. We developed a new, model-based implementation of the previously introduced focal-beam analysis method. The method's objective is to provide quantitative insight into the combined influence of acquisition geometry, overburden structure, and migration operators on image resolution and angle-dependent amplitude accuracy. This is achieved by simulation of migrated grid-point responses using focal beams. Note that the seismic response of any subsurface can be composed of a linear sum of grid-point responses. The focal beams have been chosen because any migration process represents double focusing. In addition, the focal source beam and focal detector beam relate migration quality to illumination properties of the source geometry and sensing properties of the detector geometry, respectively. Wave-equation modeling ensures that frequency-dependent effects in the seismic-frequency range are incorporated. We tested our method by application to a 3D salt model in the Gulf of Mexico. Investigation of well-sampled, all-azimuth, long-offset acquisition geometries revealed fundamental illumination and sensing limitations. Further results exposed the shortcomings of narrow-azimuth data acquisition. The method also demonstrates how acquisition-related amplitude errors affect seismic inversion results.
\end{abstract}

\section{INTRODUCTION}

A seismic survey should be designed so that the recorded data can lead to a high-quality subsurface image and accurate inversion re- sults. This notion is reflected by the seismic value chain (Berkhout, 2004) displayed in Figure 1. The seismic value chain illustrates the procedure of seismic exploration in a conceptual way. Three nodes in the chain, coupled by a double loop, represent seismic acquisition, structural imaging, and reservoir characterization. Insight is provided into interactions between these nodes. The forward path, formed by right-facing arrows, tells that acquisition geometry can introduce imaging artifacts that have to be taken into account in imaging and characterization - the acquisition geometry determines the accuracy of the imaging and characterization result. The backward path indicates that imaging and characterization should impose requirements on acquisition to realize the desired accuracy: the required imaging and characterization accuracy determine the acquisition geometry. Here, we follow the forward path in the seismic value chain. We want to assess the effect of acquisition on the image and on the reservoir characterization.

Traditionally, attributes of image quality are obtained by common-midpoint (CMP) analysis. This analysis is valid for a homogeneous or horizontally layered (1D) earth. Consequently, the influence of 2D and 3D inhomogeneities of the subsurface on the wavefields is not taken into account. However, practice indicates that downgoing and upgoing wavefields are significantly influenced by complex geology. Therefore, subsurface inhomogeneity needs to be considered in the acquisition analysis. We can do this by using the same subsurface model that is required in migration.

For this kind of analysis, ray tracing applied often is for a given source and detector geometry. Attributes are obtained from raypath information: common-reflection-point (CRP) analysis replaces the CMP analysis. Counting the number of rays (the hit count) in each CRP bin yields attributes of fold, offset, and azimuth that are related to the CRPs at the target rather than to the CMPs at the surface. Slawson et al. (1994), Muerdter and Ratcliff (2001a), Campbell et al. (2002), and Chang et al. (2002) provide examples of this approach. Dong et al. (2005) use 2D wave-equation modeling in addition to ray tracing to compute the energy distribution at the target.

Manuscript received by the Editor 21 November 2007; revised manuscript received 5 April 2008; published online 17 September 2008.

${ }^{1}$ Formerly Delft University of Technology, Delft, Netherlands; presently Chevron Energy Technology Company, San Ramon, California, U.S.A. E-mail: edith@chevron.com.

${ }^{2}$ Delft University of Technology, Delft, Netherlands. E-mail: G. Blacquiere@tudelft.nl; A. J. Berkhout@tudelft.nl.

(C) 2008 Society of Exploration Geophysicists. All rights reserved. 
Carrying out a full simulation of the seismic experiment and migrating the obtained synthetic seismic data is a computationally intensive way to obtain direct measures for image quality at the target. Image quality is visible in the migration result. However, there are more efficient ways to obtain the same information without this sequence of modeling and migration. The effect of acquisition geometry on the spatial resolution of a seismic image can be evaluated by determining the range of wavenumbers at the target (Beylkin, 1985). Vermeer (1999) investigates and applies this for a homogeneous subsurface.

For an inhomogeneous subsurface, the range of wavenumbers can be determined either with ray tracing (Gibson and Tzimeas, 2002; Lecomte and Pochon-Guerin, 2005) or wave-equation-based propagators (Toxopeus et al., 2003; Xie et al., 2006). These methods do not require a full forward simulation and a full migration. Often a target-oriented approach is followed: sought-after attributes are computed for one or more target boundaries rather than for the full subsurface volume of the macro model. Additionally, simulated Kirchhoff migration amplitudes can be calculated, as described by Laurain et al. (2002). Application of this method is demonstrated by Moldoveanu et al. (2003) and Brink et al. (2004). Because computa-

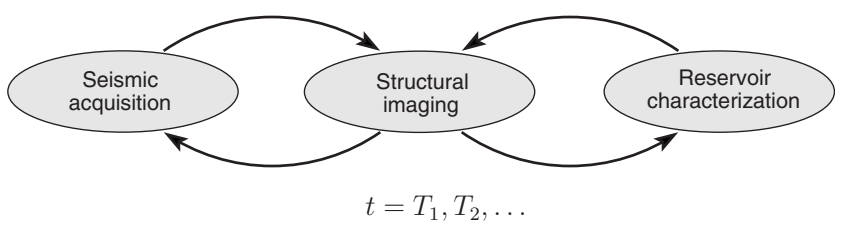

Figure 1 . The seismic value chain. $T_{1}, T_{2}, \ldots$ denote subsequent vintages of data in time-lapse seismic experiments (Berkhout, 2004). tional power is increasing, it is expected that methods based on the full sequence of 3D modeling and migration will gain interest (e.g., Jurick et al., 2003; Regone, 2006). Nevertheless, this sequence is currently too laborious to be used for an acquisition design procedure because that requires an iterative application.

We present a method based on the previously developed focalbeam-analysis concept (Berkhout et al., 2001; Volker et al., 2001). It makes use of the fundamental property that the seismic response of any subsurface can be decomposed into grid-point responses. We illustrate that grid-point responses can be analyzed separately. Our new, model-based implementation of the method uses wave-equation modeling, incorporating frequency-dependent effects in the seismic frequency range. This implementation enables accurate analysis for complex subsurfaces. It is a target-oriented method with a direct link to migration and inversion.

Use of focal beams has the advantage that the influence of source geometry and detector geometry can be separately assessed. Thus, we obtain knowledge about both target illumination by the source distribution and target sensing by the receiver distribution. Combining this information yields knowledge about the resolution of migrated data and about the accuracy of angle-dependent amplitude information. The latter can be used to show how acquisition-related amplitude errors affect seismic inversion results. Note that the source radiation pattern and receiver sensitivity pattern can be included. Figure 2 gives a diagrammatic overview of the method. It represents both the analysis part of the method (covered here) and its incorporation in an iterative design scheme. Only the primary signal is addressed in this method; coherent and incoherent noise are beyond the scope of this paper.

We illustrate our method by application to a 3D salt model in the Gulf of Mexico. Investigation of all-azimuth long-offset acquisition geometries reveals fundamental illumination limitations. Further results expose the poor illumination properties of conventional marine acquisition.

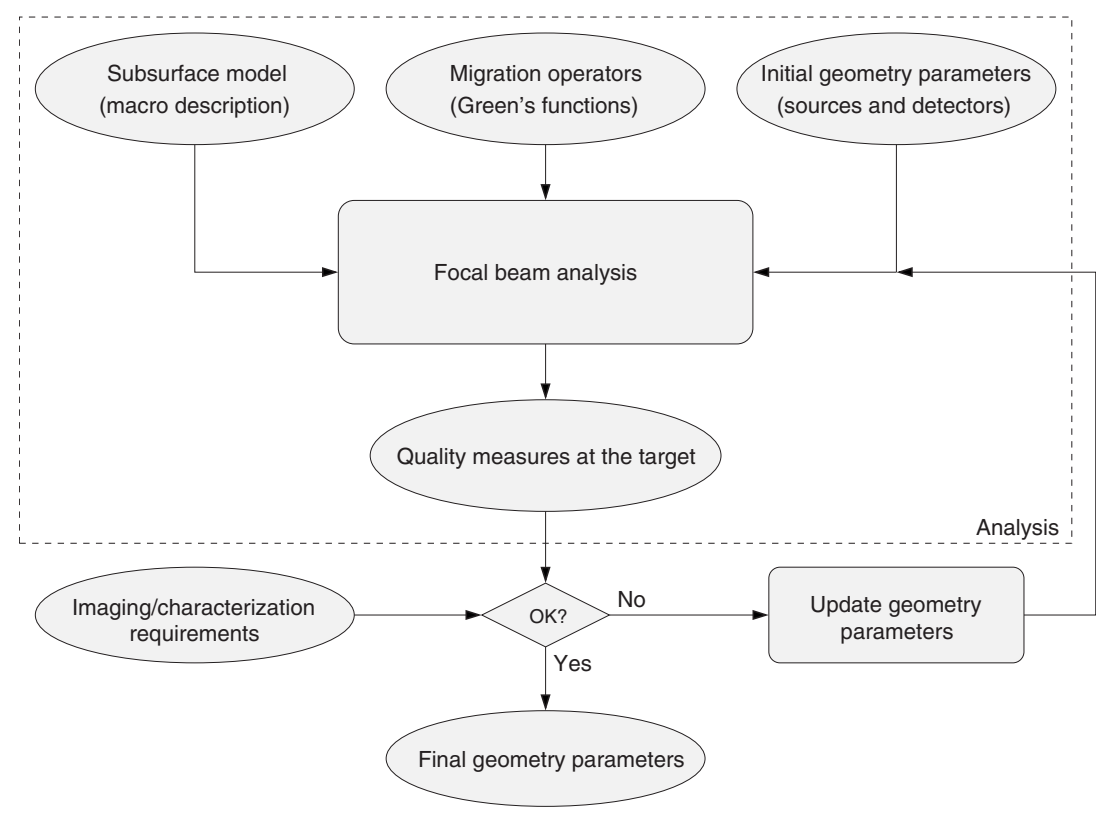

Figure 2. Focal beam analysis in an integrated approach to acquisition design (concerning primary reflected waves).

\section{METHOD}

Theoretical considerations of this method are covered largely by Berkhout et al. (2001). We will summarize the main theoretical formulations here. Additional theory is provided in Appendices $\mathrm{A}$ and $\mathrm{B}$. For further details, we refer the reader to Berkhout et al. (2001) and van Veldhuizen (2006).

\section{Forward model and decomposition into grid-point responses}

The focal-beam-analysis method originates from the imaging by double focusing concept (Berkhout, 1982, 1997). This can be explained using the WRW model, describing seismic data in terms of matrix operators in the frequency domain (Berkhout, 1982). In this model, each monochromatic component $\mathbf{P}\left(z_{0}, z_{0}\right)$ of the primary wavefield that is recorded at the surface $z_{0}$, can be described in the space-frequency domain as 


$$
\begin{aligned}
\mathbf{P}\left(z_{0}, z_{0}\right)= & \mathbf{D}\left(z_{0}\right) \sum_{m=1}^{M}\left[\mathbf{W}\left(z_{0}, z_{m}\right)\right. \\
& \left.\times \mathbf{R}\left(z_{m}, z_{m}\right) \mathbf{W}\left(z_{m}, z_{0}\right)\right] \mathbf{S}\left(z_{0}\right) .
\end{aligned}
$$

Depth levels $z_{m}$ are levels where the waves are reflected. The designation "WRW model" stems from the two matrix operators $\mathbf{W}$, and matrix operator $\mathbf{R}$, which are explained below. Figure 3 shows a schematic representation of the model. Lateral coordinates $x$ and $y$ and frequency $\omega$ have been left out for convenience. The matrix operators in equation 1 have the following meaning:

- $\mathbf{S}\left(z_{0}\right)$ is the source matrix, containing amplitude and phase of the source wavelet at the frequency under consideration. One column represents one source array. Each column in the matrix corresponds to a lateral location in space.

- $\mathbf{W}\left(z_{m}, z_{0}\right)$ is a forward-wavefield-propagation matrix. Each column contains a discretized Green's function describing wave propagation from one point (one lateral location) at surface $z_{0}$ to many points at depth level $z_{m}$. Note that any one-way wave-propagation method could be used to compute this matrix, depending on required accuracy and available computer resources.

- $\mathbf{R}\left(z_{m}, z_{m}\right)$ is the reflectivity matrix, describing conversion of an incident wavefield into a reflected wavefield, as will be explained further below.

- $\mathbf{W}\left(z_{0}, z_{m}\right)$ is a forward-wavefield-propagation matrix. Each column contains a discretized Green's function describing wave propagation from one point at the reflection level $z_{m}$ to many points at the surface $z_{0}$.

- $\mathbf{D}\left(z_{0}\right)$ is the detector matrix, containing the detector wavelet and the locations of detector arrays. One row represents one detector array at a certain lateral location in space.

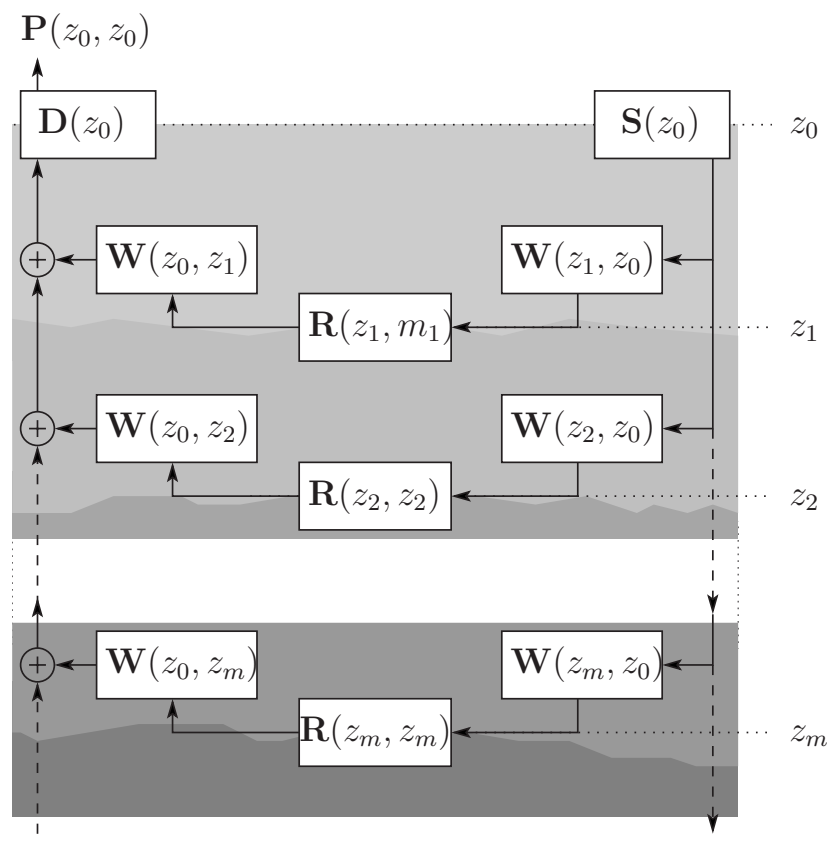

Figure 3. The WRW model, after the formulation of equation 1. For each reflection, a WRW term is added to the total expression for the measured pressure $\mathbf{P}\left(z_{0}, z_{0}\right)$.
It follows that the columns of data matrix $\mathbf{P}\left(z_{0}, z_{0}\right)$ correspond to surface locations for sources, and rows correspond to surface locations for detectors. Therefore, one column of this data matrix represents a common-source gather (shot record) and one row represents a common-receiver gather. Other data gathers, such as CMP gathers and/or common-offset gathers, also can be identified in the data matrix. This equation is valid for stationary acquisition geometries and stationary parts of nonstationary acquisition geometries; i.e., in $\mathbf{S}\left(z_{0}\right)$, all sources are included that shoot into the same range of detector locations, represented by $\mathbf{D}\left(z_{0}\right)$. Furthermore, this model applies to both 3D and 2D space. Our examples deal with a 3D subsurface. Figure 4 gives a simplified example of a data matrix for towedstreamer acquisition.

Note that the WRW model in the form of equation 1 is one-way and does not contain multiple reflections, unless explicitly included in the formulation. Surface multiples can be included in the model by creating a feedback loop (Berkhout, 1982).

Each column of the reflectivity matrix $\mathbf{R}\left(z_{m}, z_{m}\right)$ in equation 1 contains the angle-dependent reflection operator of one grid point at reflecting interface $z_{m}$, where $z_{m}=z_{m}(x, y)$. The operator represents the angle-dependent reflectivity relation between the incident wavefield at the grid point under consideration (one lateral location at $z_{m}$ ) and the reflected wavefield in the area around that grid point (all lateral locations at $z_{m}$ ). A linear Radon transform of such an operator shows the individual reflection coefficient for each angle of incidence. De Bruin et al. (1990) explain the reflectivity matrix extensively. A special version of the reflectivity matrix containing only one nonzero column $k$ is called $\delta_{k} \mathbf{R}$, where $k$ indicates lateral location $(x, y)_{k}$ of the incident wavefield. The total reflectivity matrix $\mathbf{R}$ can be written as a sum of such grid-point reflectivity matrices according to:

$$
\mathbf{R}\left(z_{m}, z_{m}\right)=\sum_{k} \delta_{k} \mathbf{R}\left(z_{m}, z_{m}\right)
$$

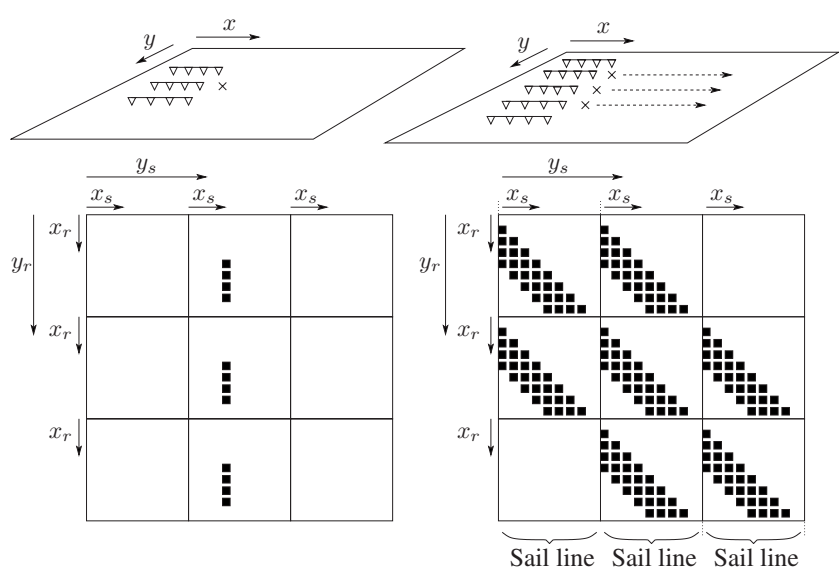

Shot record

Three sail lines

Figure 4. Data matrix in three spatial dimensions for a towedstreamer configuration. The data matrix has two dimensions: one related to the source locations (columns) and one related to the receiver locations (rows). All $x$-coordinates related to one $y$-coordinate are contained in a submatrix. The subscript $s$ refers to source, whereas the subscript $r$ refers to receiver. The top panels show the configuration of sources and detectors at the acquisition surface, where sources are denoted by $\times$ and receivers are denoted by $\nabla$. 
Figure 5 illustrates equation 2 . In the case of an angle-independent unit reflector, $\mathbf{R}\left(z_{m}, z_{m}\right)$ becomes a unit matrix and $\delta_{k} \mathbf{R}\left(z_{m}, z_{m}\right)$ has only one nonzero element: its value is one at row $k$ and zero elsewhere. This means that the incident wavefield at grid-point $k$ contributes to the reflected wavefield at gridpoint $k$ only. Equation 2 gives the opportunity to relate grid-point properties to full reflector properties.

Considering equations 1 and 2, we see that the recorded reflection response can be decomposed into (and built from) grid-point responses:

$$
\mathbf{P}\left(z_{0}, z_{0}\right)=\sum_{k} \delta_{k} \mathbf{P}\left(z_{0}, z_{0}\right) .
$$

In the remainder of this paper, we will consider grid-point responses, knowing that they represent one component of the reflector and that any reflector can be built from a collection of these responses.

More specifically, we will use angle-independent reflection properties at any grid point we want to analyze in our modeling. We want to find the angle-dependent acquisition footprint, irrespective of any angle-dependent subsurface properties.

By using angle-independent subsurface reflectivity in modeling, we can be sure that any angle-dependent amplitude effects must have been introduced by the acquisition geometry. Consequently, angle-dependent grid-point responses in our modeling are replaced by angle-independent grid-point responses, i.e., by point diffractors.

\section{Focal beams}

Describing migration as a double-focusing process (Berkhout, $1982,1997)$ is the next step leading to the focal beam analysis method. The goal of migration is to retrieve the reflectivity from surface seismic data. For this purpose, wavefield-propagation effects need to be removed, i.e., the $\mathbf{W}$ operators of equation 1 should be eliminated. This is achieved by applying migration operators or focusing operators $\mathbf{F}$ :

$$
\begin{aligned}
\mathbf{P}_{i j}\left(z_{m}, z_{m}\right)= & \mathbf{F}_{i}^{\dagger}\left(z_{m}, z_{0}\right) \mathbf{P}\left(z_{0}, z_{0}\right) \mathbf{F}_{j}\left(z_{0}, z_{m}\right) \\
= & {\left[\mathbf{F}_{i}^{\dagger}\left(z_{m}, z_{0}\right) \mathbf{D}\left(z_{0}\right)\right] \mathbf{W}\left(z_{0}, z_{m}\right) \mathbf{R}\left(z_{m}, z_{m}\right) \mathbf{W}\left(z_{m}, z_{0}\right) } \\
& \times\left[\mathbf{S}\left(z_{0}\right) \mathbf{F}_{j}\left(z_{0}, z_{m}\right)\right] .
\end{aligned}
$$

Subscript $i$ indicates that the $i$ th row of the matrix is selected. A row vector is marked by a dagger symbol $(\dagger)$. Subscript $j$ indicates that the $j$ th column of the matrix is selected. At the detector side, operator $\mathbf{F}_{i}^{\dagger}\left(z_{m}, z_{0}\right)$ removes the wave propagation between the reflection and detection levels, $\mathbf{W}\left(z_{0}, z_{m}\right)$. Together, $\mathbf{F}_{i}^{\dagger}\left(z_{m}, z_{0}\right)$ and $\mathbf{D}\left(z_{0}\right)$

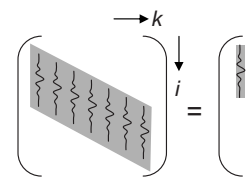

$\mathbf{R}$
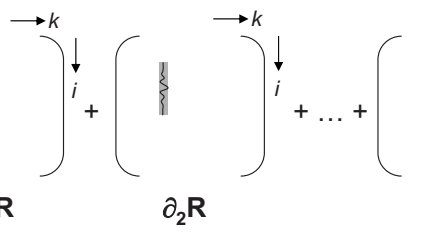

Figure 5. Reflectivity matrix $\mathbf{R}$ can be composed of a linear sum of grid-point reflectivity matrices $\delta_{k} \mathbf{R}$. Each matrix $\delta_{k} \mathbf{R}$ contains the angle-dependent reflection properties at location $k$ along the reflector. Note that index $k$ indicates the lateral location of the incident wavefield and index $i$ indicates the lateral location of the reflected wavefield. represent a focusing detector array. A perfect migration would require

$$
\mathbf{F}_{i}^{\dagger}\left(z_{m}, z_{0}\right) \mathbf{D}\left(z_{0}\right) \mathbf{W}\left(z_{0}, z_{m}\right)=\mathbf{I}_{i}^{\dagger}\left(z_{m}\right) .
$$

$\mathbf{I}_{i}^{\dagger}\left(z_{m}\right)$ is a row vector out of identity matrix $\mathbf{I}\left(z_{m}\right)$, where the $i$ th element is the only nonzero element. Equation 5 implies that the detector geometry and the focusing operator are designed such that they allow perfect removal of wave-propagation effects. We define the focal detector beam $\mathbf{D}_{i}^{\dagger}\left(z_{m}, z\right)$ as

$$
\mathbf{D}_{i}^{\dagger}\left(z_{m}, z\right)=\mathbf{F}_{i}^{\dagger}\left(z_{m}, z_{0}\right) \mathbf{D}\left(z_{0}\right) \mathbf{W}\left(z_{0}, z\right),
$$

with its focus point at $z=z_{m}$. Note that the focal beam is defined at all depth levels $z$.

A similar train of thought applies to the source side of the seismic experiment. Ideally,

$$
\mathbf{W}\left(z, z_{0}\right) \mathbf{S}\left(z_{0}\right) \mathbf{F}_{j}\left(z_{0}, z_{m}\right)=\mathbf{I}_{j}\left(z_{m}\right),
$$

in which $\mathbf{I}_{j}\left(z_{m}\right)$ is a column vector where the $j$ th element is the only nonzero element. $\mathbf{S}\left(z_{0}\right) \mathbf{F}_{j}\left(z_{0}, z_{m}\right)$ represents a focusing source array, which should be designed to allow perfect removal of wave-propagation effects on the source side. We define the focal source beam $\mathbf{S}_{j}\left(z, z_{m}\right)$ as

$$
\mathbf{S}_{j}\left(z, z_{m}\right)=\mathbf{W}\left(z, z_{0}\right) \mathbf{S}\left(z_{0}\right) \mathbf{F}_{j}\left(z_{0}, z_{m}\right),
$$

with its focus point at $z=z_{m}$

Physically, applying the two focusing operators $\mathbf{F}$ in equation 4 represents two focusing steps. For structural imaging, the two focusing operators are applied for the same subsurface grid point $(i=j)$. Alternatively, $i$ can be varied around $j$ (or vice versa), to obtain angle-dependent reflection information. It should be noted that this description of migration as a double-focusing process is valid for any migration method: in Kirchhoff migration and wavefield-continuation migration methods, there are always two (recursive) weighted summation steps over spatial coordinates.

Imaging by double focusing for all subsurface grid points, i.e., for a range of lateral locations and depth levels, yields a migrated seismic volume. Figure 6 illustrates the focal beams obtained for a twodimensional homogeneous medium. It also illustrates the process of migration by double focusing for a grid-point response. The focal source beam was computed for sources at lateral locations ranging from $0 \mathrm{~m}$ to $1000 \mathrm{~m}$ and the focal detector beam for detectors at lateral locations ranging from $1300 \mathrm{~m}$ to $2300 \mathrm{~m}$. Alternative (computationally useful) formulations of focal beams are given in Appen$\operatorname{dix}$ A.

In practice, the acquisition geometry usually does not allow perfect focusing. The focal detector beam shows how the detector geometry affects focusing, and the focal source beam shows how the source geometry affects focusing. This offers an opportunity to judge and adjust the configuration of sources and detectors separately. Moreover, if the focusing operators are not perfect migration operators, the focal beams also show migration-operator artifacts. This means that focal beam analysis also can contribute to the validation of migration algorithms.

Similar to retrieving angle-dependent information from the $\mathbf{R}$ matrix in the Radon domain (de Bruin et al., 1990), a plane-wave decomposition of focal beams by means of a linear Radon transform reveals the angles for which the target point is illuminated (source beam) or sensed (detector beam). The Radon-transformed focal source beam shows angles of incidence at the target point as a result 
of the chosen source geometry, and the focal detector beam shows take-off angles at the target point that can be sensed by the detectors.

Focal beams are inspected at zero time (image time) in the spatial domain and at zero intercept time in the Radon domain. Note however that the focal beams are formulated as a full wavefield at the target level (in terms of their monochromatic components). This allows further analysis. First, multiplication of the focal beams yields the double-focused wavefield (see Figure 6). This is the resolution function or point-spread function of the grid point under consideration. Next, multiplication of the Radon-transformed focal beams yields the AVP (amplitude versus ray parameter) imprint. In this way, source-distribution illumination angles are combined with detectordistribution sensing angles. Therefore, the AVP imprint shows how angle-dependent reflection amplitudes are affected by the acquisition geometry, overburden structure, and migration operators. Theoretical formulation of the resolution function and the AVP imprint are given in Appendix B.

Instead of a horizontal plane around the target point, a dipping plane can be considered (van Veldhuizen, 2006). The local reflector dip can be incorporated in the focal beams easily by a ray-parameter shift in the Radon domain. This is done after the focal beams have been computed. This means that, with little extra computation effort, the results for a range of reflector dips can be evaluated.

\section{Implementation}

To apply focal beam analysis, equations A-3 and A-4 (Appendix A) need to be evaluated. These are computationally more efficient (but equivalent) versions of equations 6 and 8 at depth level $z_{m}$, as noted in Appendix A. To simulate forward wave propagation (operators $\mathbf{W}$ ), a method should be chosen that models the true wave propagation as accurately as possible. For focusing (operators $\mathbf{F}$ ), the method should correspond to the migration algorithm that is applied in practice.

The method's efficiency depends greatly on the user's choice of wave propagator, which can range from eikonal solvers (Vidale, 1988 ) to full 3D elastic finite-difference methods (Virieux, 1986). Combined with available computer resources, this choice determines the number of target points that can be evaluated within a given computation time. Thus the user has the option to trade off quantity, efficiency, and quality. We have chosen to use recursive depth extrapolation by convolutions in the space-frequency domain, or $x y-\omega$ extrapolation (Holberg, 1988; Blacquière et al., 1989), with a weighted least-squares-operator optimization (Thorbecke et al., 2004). We use this method for a number of reasons: frequency-dependent propagation effects can be incorporated in the results; the one-way wave-equation method is more efficient than two-way wave-equation methods; accuracy is high in 3D complex media; and the method can be implemented in a straightforward manner. In the implementation of focal beam analysis as used here, the $x y-\omega$ extrapolation is applied both for simulation of forward wave propagation and for focusing.

Note that multiple scattering and transmission coefficients are not included in one-way wave-equation methods. It is not desired to include multiple scattering because the method presented here aims at analysis of the primary-reflected-wave content of the data. However, if it is desired to include transmission coefficients, a more complex (and more time-consuming) modeling method is required.
Computation of focal beams consists of the following steps:

- Select a grid point at the target.

- Simulate the angle-independent grid-point response by forward wave propagation from this grid point to the acquisition level $\left(\mathbf{W}_{j}^{\dagger}\left(z_{m}, z_{0}\right)\right.$ and $\mathbf{W}_{j}\left(z_{0}, z_{m}\right)$ in equations A-3 and A-4). This yields a Green's function.

- Apply the acquisition geometry. Traces corresponding to source
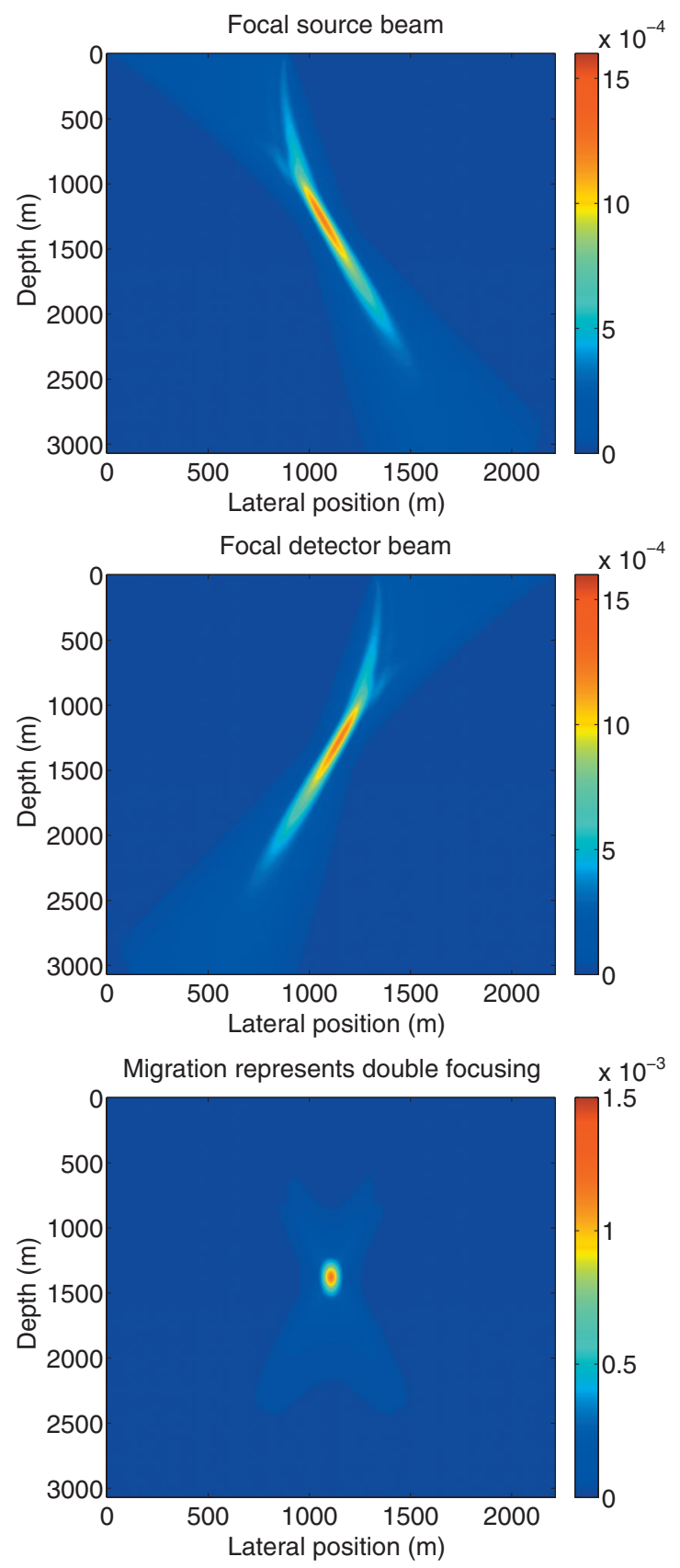

Figure 6. Focal source beam (top) and focal detector beam (middle) visualized for all depth levels. The double focusing result (bottom) is obtained by multiplying the focal source beam and the focal detector beam for each frequency component. Migration corresponds to double focusing for all subsurface grid points followed by the imaging step (summation over all frequencies). The colors indicate the absolute amplitudes on a linear scale. 
locations are selected for computation of the focal source beam. To compute the focal detector beam, traces that correspond to receiver locations are selected.

- Apply focusing from the acquisition level to a volume around the target point. For focusing on the source side, use operator $\mathbf{F}\left(z_{0}, z_{m}\right)$ in equation A-4. For focusing on the detector side, use operator $\mathbf{F}\left(z_{m}, z_{0}\right)$ in equation A-3.

The considered volume around the target point should have a sufficient extent to include the locations where the main imaging artifacts occur for this grid-point location (e.g., spatial aliasing).

Acquisition-geometry trace selection and the focusing step are repeated for all stationary parts of the acquisition geometry, meaning that only sources and detectors are included, between which there is wave propagation in the survey. Thus the method is most efficient for
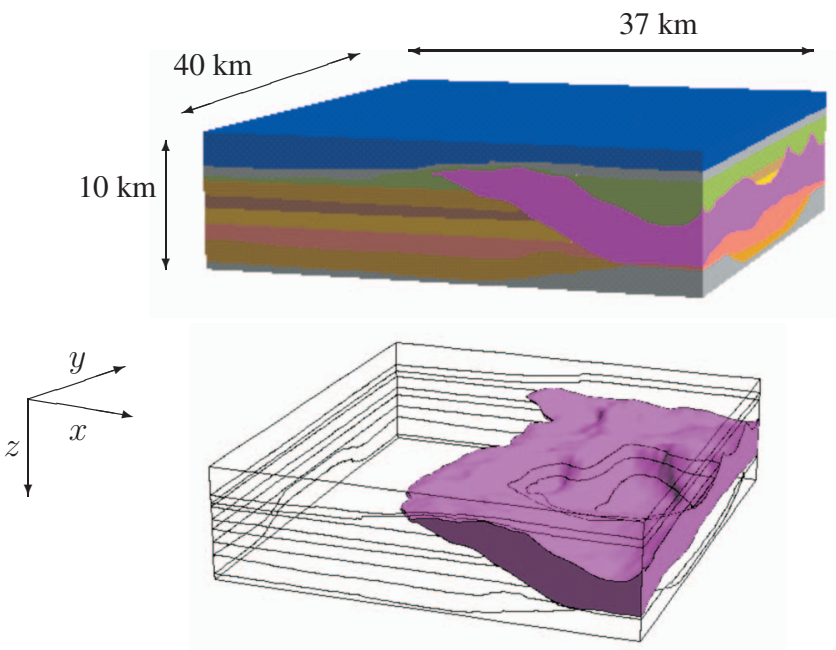

Figure 7. Ziggy model. The top picture shows the full model with indications of the dimensions; the bottom picture shows a wire frame of the model with only the salt structure in it.

a)

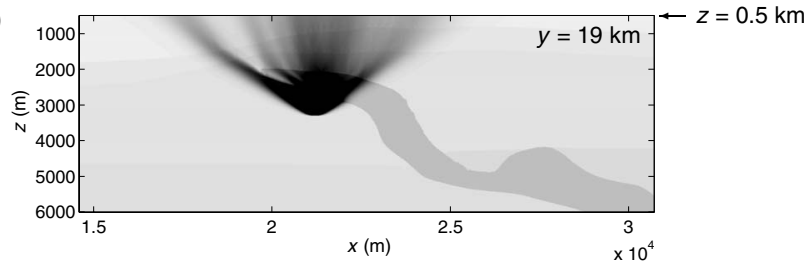

b)

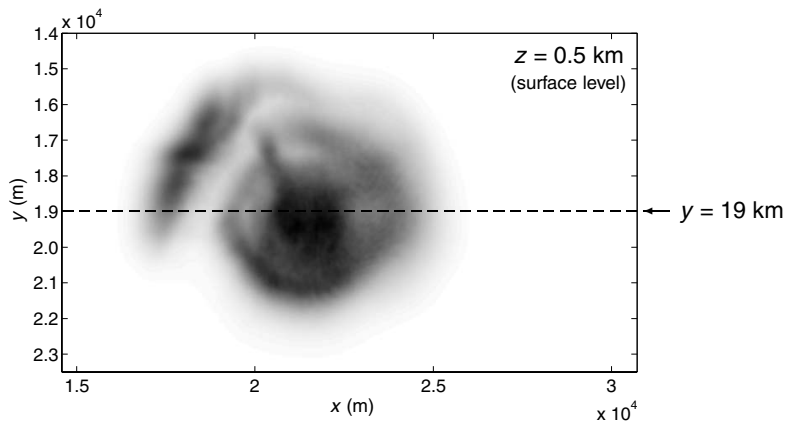

Figure 8. 2D slices of a 3D energy beam for upward wave propagation from a grid point at $3300 \mathrm{~m}$ depth. (a) The structure of the salt body causes variations in energy level at the surface, as observed in (b). The energy is displayed on a linear scale. acquisition geometries with large stationary parts, like $\mathrm{OBC}$ and land geometries, and least efficient for geometries with small or no stationary parts. An example of the latter is a towed-streamer marine geometry, in which the detector spread is constantly moving (no two sources shoot into the same range of detector locations).

\section{EXAMPLE}

Illustrations in this section show the influence of an irregularly shaped salt structure on illumination strength and detection sensitivity — and the consequent AVP imprint — for subsalt target points. We treat the following aspects:

- The effect of a complex salt structure on the wavefield that propagates upward from an exploding grid point

- Location dependence of beams and imprint

- Frequency dependence of beams and imprint

- The effect of conventional towed streamer marine geometries on subsalt image quality

The emphasis is on the angle-dependent amplitude information. It is known that this information is highly affected and often irretrievable in subsalt areas. Therefore, it is useful to investigate what information is retrievable potentially with a well-sampled, all-azimuth, long-offset acquisition geometry, and then to investigate what information is lost by using a conventional marine geometry.

\section{Macro model}

The Ziggy model is a Gulf-of-Mexico salt model developed by SMAART JV (Subsalt Multiple Attenuation and Reduction Team, 1998-2002, originally a joint venture of BHP, BP Amoco, Chevron, Mobil, and Texaco. [See http://www.delphi.tudelft.nl/SMAART]). It is a model with several layers that are more or less flat, and a complex salt structure. Total dimensions of the model are approximately $37 \mathrm{~km}(x) \times 40 \mathrm{~km}(y) \times 10 \mathrm{~km}(z)$. The model is illustrated in Figure 7. Velocity is $4850 \mathrm{~m} / \mathrm{s}$ in the salt body and $1950 \mathrm{~m} / \mathrm{s}$ in the water-bottom layer. Velocities in other layers vary from $2250 \mathrm{~m} / \mathrm{s}$ to $3000 \mathrm{~m} / \mathrm{s}$. Grid spacing in the model is $25 \mathrm{~m}$ in the lateral direction and $10 \mathrm{~m}$ in the depth direction.

The maximum frequency used in the following illustrations is $30 \mathrm{~Hz}$. The extrapolation operators have a length of 25 grid points in both lateral directions $x$ and $y$, and they are optimized for a maximum propagation angle of $60^{\circ}$. For larger angles, amplitude and phase of the operators go smoothly to zero (Thorbecke et al., 2004).

\section{Subsalt target points: Forward modeling and focal beams}

Figure 8 depicts upward propagating energy from an exploding grid point. The target point is located at $(x, y, z)=(21.2,19,3.3) \mathrm{km}$, and the wavefield is propagated upward to a depth of $500 \mathrm{~m}$, which will be considered the surface. The top $500 \mathrm{~m}$ of the water layer are not considered in this case. Figure 8 illustrates how salt body structure causes variations in surface energy level. Several effects play a role: strong refractions and postcritical incidence at the base of salt for relatively small angles with respect to the vertical. This is caused by the high velocity contrast and the dip of the base of salt. Furthermore, the extrapolation operators have been designed for a maximum wave-propagation angle of $60^{\circ}$.

It is unlikely that energy critically reflected from the base of salt would reach the acquisition surface in practice. Loss of that energy 
results in missing information in the imaged grid-point response. This is visible in Figure 9, which illustrates the result of inversely extrapolating the energy at the surface back to the target level, i.e., a focal beam for a full 3D acquisition geometry. This situation represents detection (or illumination) of the target point by detectors (or sources) densely covering the complete acquisition surface area. This means that all azimuths are included: an ultimate rich-azimuth or wide-azimuth situation. Offsets range up to $5000 \mathrm{~m}$ in the $y$-direction and up to $8000 \mathrm{~m}$ in the $x$-direction, as can be observed in Figure $8 \mathrm{~b}$ (the surface area depicted here is also the acquisition area). Figure $8 \mathrm{~b}$ also illustrates that the main signal seems to be well captured within this aperture.

The focal beam, transformed to the Radon domain, reveals the angles and azimuths at the target from which this target point can be illuminated or detected. Figure 9a depicts a range of angles and azimuths for which the amplitude is fairly uniform, and an additional high-amplitude area for high angles (top left). The former represents the portion of the wavefield that has traveled through the salt. For that part of the wavefield, an angle limitation arises for high angles, because of the limited migration-operator angle: the migration operators suppress the part of the wavefield propagating in the salt with angles higher than about $60^{\circ}$. The second high-amplitude area (top left) represents waves traveling past the edge of salt, which also can be observed in Figure 8a. These waves have not been subjected to strong refractions at the salt boundaries. The gap between the two high-amplitude regions is caused partly by the angle limitation of the migration operators, and it is caused partly by the postcritical wave incidence at the base of salt. Note that, because a one-way modeling method is used, scattered energy is not modeled and therefore is not visible in Figure 8.

The amplitudes of two cross-sections of the focal beam, which are indicated by dashed lines in Figure 9a, are illustrated in Figure 9b and c. Bottom graphs depict amplitudes as a function of ray $p$ (just as in Figure 9a), and the top graphs show amplitudes as a function of ray angle. A dashed blue line indicates the $-20 \mathrm{~dB}$ amplitude level. The decrease of amplitudes for high angles is caused by angle limitation of the migration operators. For positive angles, this decrease starts at smaller angles than for negative angles. This is caused by the refractions at salt boundaries. The amplitude gap between approximately $-25^{\circ}$ and $-35^{\circ}$, caused by postcritical wave incidence, is clearly visible as well.

For comparison, focal beams for two different subsalt target points are depicted in Figure 10. Note that a 3D area is considered in both cases, but for display purposes single slices of the considered areas are illustrated in Figures 10a and b. In the first case, the target point is located at $(x, y, z)=(25,31,6) \mathrm{km}$, below a rather flat area of the base of salt (target point 1). In the second case, the target point is located at $(x, y, z)=(23,19,6) \mathrm{km}$, right below a steeply dipping part of the salt (target point 2).

Parameters for both cases are slightly different. For target point 1 , the modeling and acquisition aperture is $18 \mathrm{~km}$ (in the $x$-direction) $\times 12 \mathrm{~km}$ (in the $y$-direction), and the frequency range under consideration is $10 \mathrm{~Hz}$ to $30 \mathrm{~Hz}$. For target point 2, lateral dimensions of the modeling and acquisition aperture are $19.2 \times 19.2 \mathrm{~km}$, and the frequency range is $2 \mathrm{~Hz}$ to $30 \mathrm{~Hz}$. However, the frequency spectrum for the range of 10 to $30 \mathrm{~Hz}$ is the same in both cases. Furthermore, to save computational effort, $800 \mathrm{~m}$ has been taken off the water layer in the computations for target point 1.

For these target points, similar effects can be observed as for the case depicted in Figures 8 and 9. For target point 1, the main effects are angle limitations because of limited aperture in the $y$-direction and the angle limitation of the migration operators. Furthermore, the amplitude is fairly uniform, which can be observed in the bottom panel of Figure 10c. For target point 2, there is again a situation where postcritical wave incidence occurs at the base of salt. In this case, because the target point is located directly below the steepest part of the base of salt, postcritical wave incidence occurs for many low angles. Note however that there is still energy present for these angles, although lower than $-20 \mathrm{~dB}$. For negative values of $p_{x}$, corresponding to low values of $x$ (left), the high amplitudes are caused by waves traveling past the edge of salt. High amplitudes for positive values of $p_{x}$, corresponding to higher values of $x$, are caused by waves that do penetrate the salt. a)

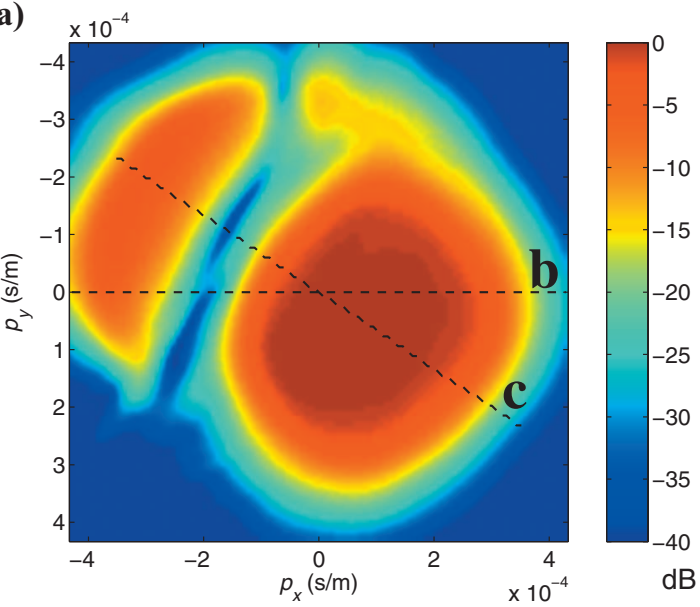

b)
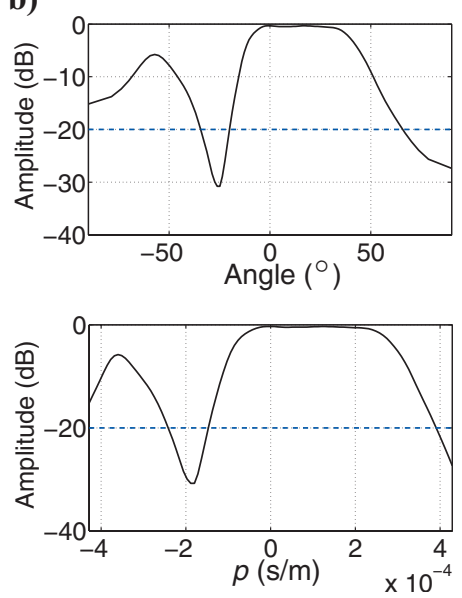

c)
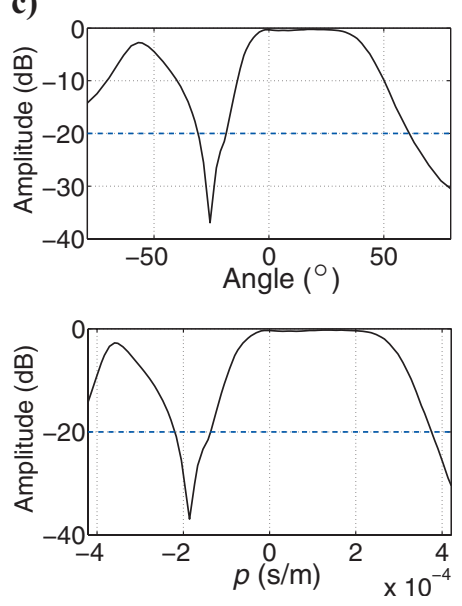

Figure 9. Focal beam for a full 3D geometry for the target point located at $(x, y, z)=(21.2,19,3.3) \mathrm{km}$, for which the upward propagating wavefield is depicted in Figure 8. Two dashed lines in (a) indicate the locations of the cross sections in (b) and (c). Note that the bottom images of (b) and (c) illustrate amplitudes as a function of ray parameter $p$, just as in (a), and the top images give amplitudes as a function of angle. The latter offers a better grasp of the angles for which the amplitude falls below the $-20 \mathrm{~dB}$ line, indicated by dashed blue lines. 
These results are consistent with the observation of areas of poor illumination in subsalt situations, as addressed, e.g., by Muerdter and Ratcliff (2001b). Illumination depends strongly on the structure of the salt.

\section{Monochromatic focal beams}

A method that operates in the seismic frequency band is advantathe seismic data, but also because frequency-dependent effects can be analyzed. Figure 11 depicts monochromatic components of the focal beams in Figure 10. These components have been normalized individually; however, in the focal beam for full bandwidth, they will have different strengths corresponding to the seismic spectrum.

It can be observed that for lower frequencies, angles extend farther beyond the maximum propagation angle for which extrapolation operators have been optimized. This occurs because it is algeous not only because the result corresponds to what is observed in

lowed in the operator optimization - as long as the wavenumber spectrum is accurate for the desired range of propagation angles and smoothly decays outside that range, to reach zero at the Nyquist wavenumber (Thorbecke et al., 2004). Because the Nyquist wavenumber is frequency independent, it corresponds to larger ray-parameter values for lower frequencies. Therefore the amplitudes for lower frequencies decay for larger ray parameters.

In addition, it can be observed that the results for higher frequencies show more detailed amplitude variations. This results from smaller wavelengths, which yield a higher resolution in the Radon domain. Moreover, if the wavelength has the same order of magnitude as irregularities of the salt boundary, diffraction occurs. This does not occur for larger wavelengths. Therefore, higher frequencies are more sensitive to irregular salt boundaries.

Note also that the illuminated-angle range around $\left(p_{x}, p_{y}\right)$ $=(1,-1) \cdot 10^{-4}$ (see Figure 10d) appears to be illuminated and detected mainly by low frequencies. As mentioned, many waves in this angle range have postcritical incidence angles. a)
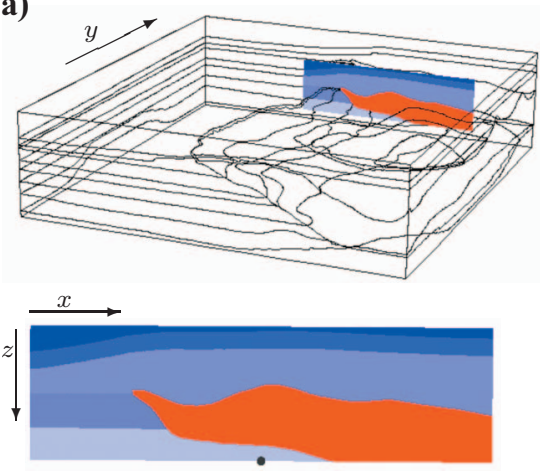

b)
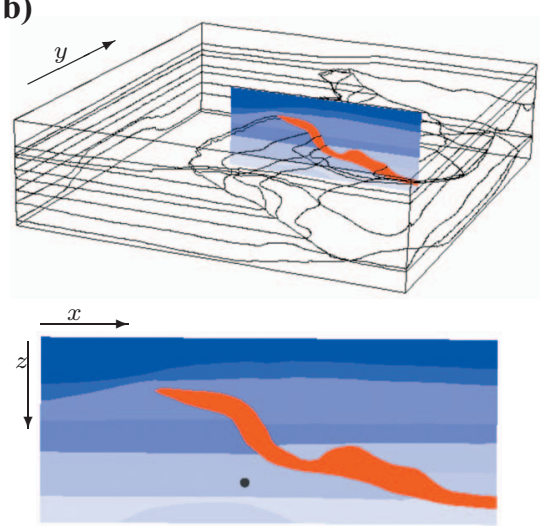

c)
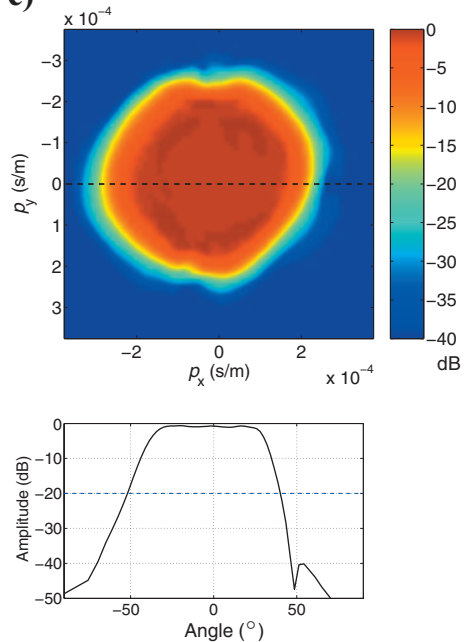

d)
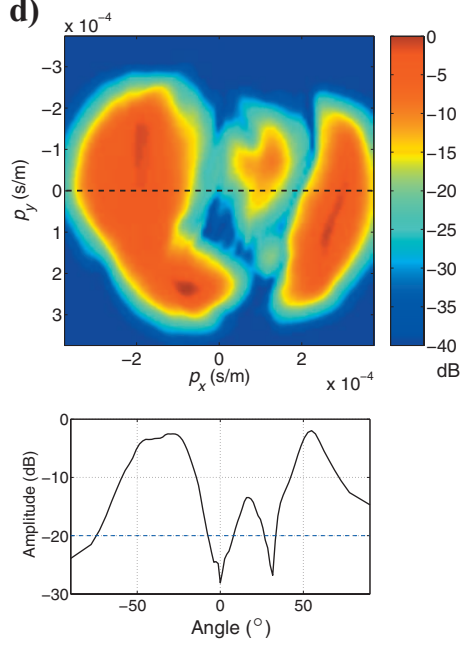

Figure 10. Focal beams in the Radon domain for a full 3D acquisition geometry for subsalt target points located at $(x, y, z)=(25,31,6) \mathrm{km}(\mathrm{left})$ and at $(x, y, z)=(23,19,6) \mathrm{km}$ (right). Parts (a) and (b) depict slices of the areas under consideration, in which the target point is indicated by a black dot. Parts (c) and (d) illustrate the corresponding focal beams. The top images in (c) and (d) show amplitude as a function of $p_{x}$ and $p_{y}$, and black dashed lines indicate $p_{y}=0$. Bottom images show amplitude for $p_{y}=0$, as a function of angle, where blue dashed lines indicate $-20 \mathrm{~dB}$.
Waves that do penetrate the salt have a low-frequency content. Once again, this results from stronger scattering of high frequencies than of low frequencies.

This means that illumination studies making use of ray tracing — based on the high-frequency approximation - give potentially a too-pessimistic view of the illumination. Shadow zones that occur in ray-tracing results (Muerdter and Ratcliff, 2001b) can be illuminated by low frequencies.

Absorption also plays a role in practice, but that aspect is not included in this modeling experiment. The same applies for elastic low-pass effects (Ziolkowski and Fokkema, 1986), because acoustic modeling is used.

\section{Lateral resolution and AVP imprint}

The focal beams given in Figures 9-11, illustrate from which angles the target point can be illuminated and detected. Figure 12 shows the result for combined illumination and detection: the resolution functions and AVP imprints. Results are depicted for the three subsalt target points considered earlier - compare Figure 12a to Figure 9 and Figure 12b and c to Figure 10c and d. Velocity is $2330 \mathrm{~m} / \mathrm{s}$ at the target point in Figure 12a. For the other two target points, which are at the same depth, velocity is $2700 \mathrm{~m} / \mathrm{s}$. In all AVP imprint displays, the maximum magnitude of $p_{x}$ and $p_{y}$ is equal to $\mathrm{P}$-wave slowness at the target point, corresponding to a $90^{\circ}$ reflection angle.

Because of the full 3D geometry, the focal detector beam and focal source beam are identical. The resolution function results from element-byelement multiplication of two identical focal beams. The ideal result would be a spatially bandlimited delta pulse, i.e., a small black dot with (weak) side lobes in the plane displayed here. The Radon-transformed focal beams are depicted in Figure 9a and Figure 10c and d. The AVP imprints 

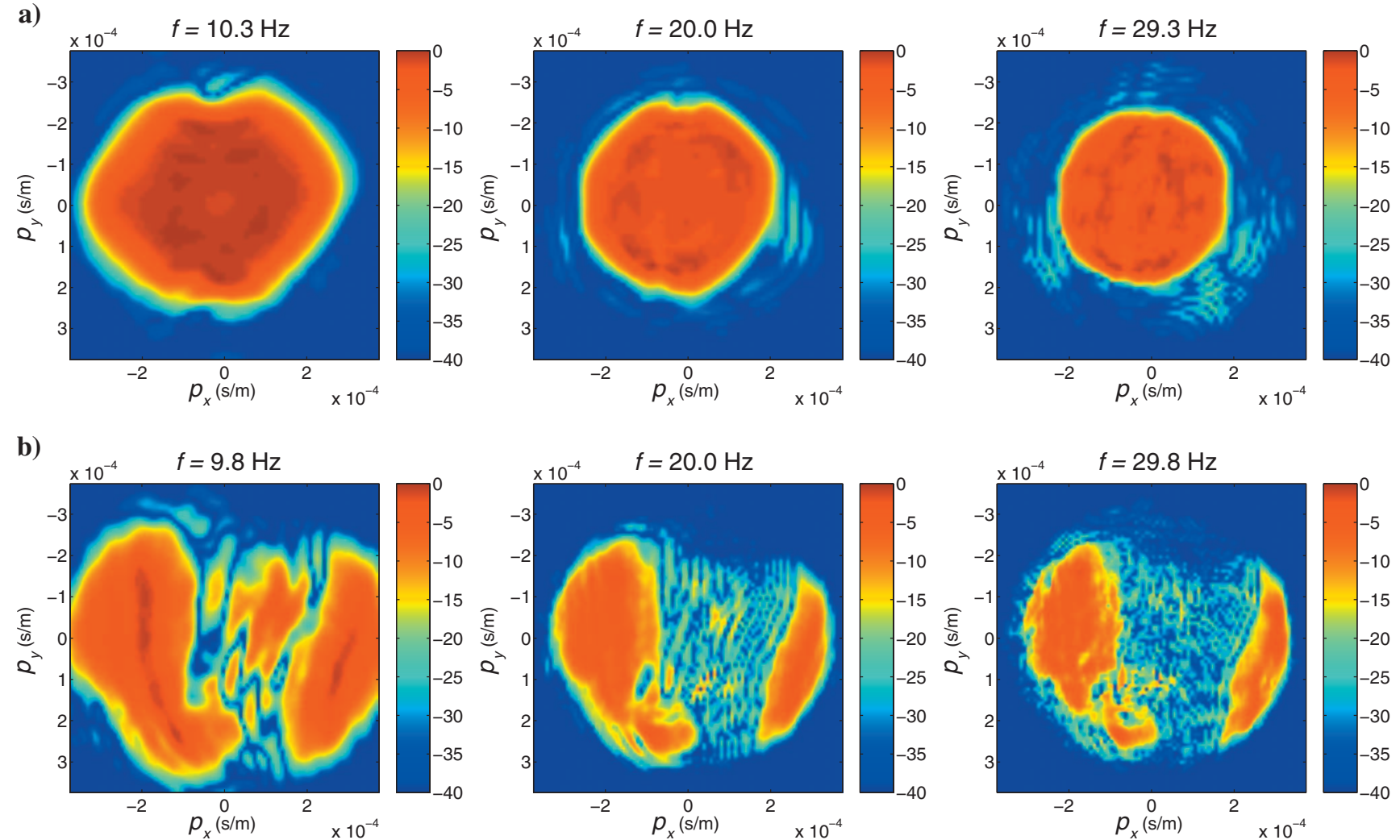

Figure 11. Monochromatic components of the focal beams in Figure 10. The frequencies are approximately $10 \mathrm{~Hz}$ (left), $20 \mathrm{~Hz}$ (middle), and $30 \mathrm{~Hz}$ (right). The focal beams have been normalized individually. The color scale unit is $\mathrm{dB}$.

a)

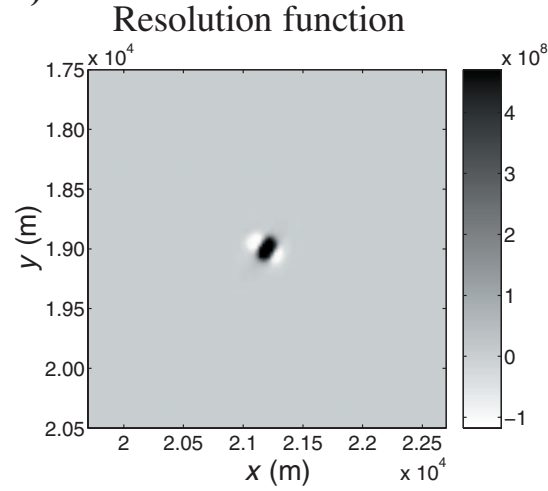

AVP imprint

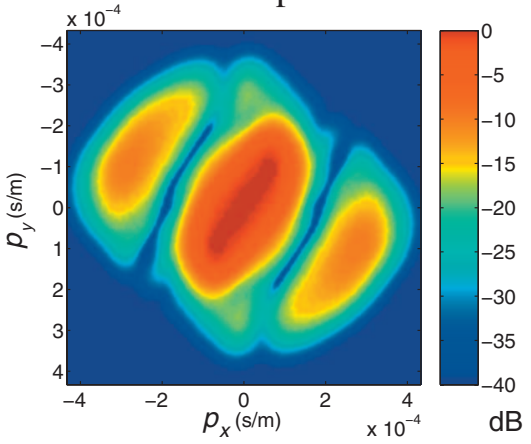

b)

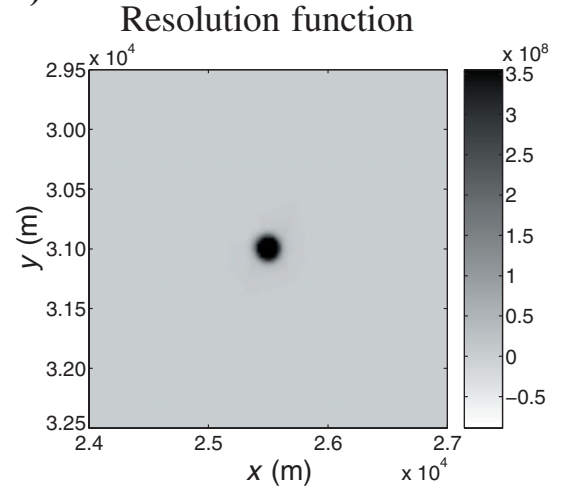

AVP imprint

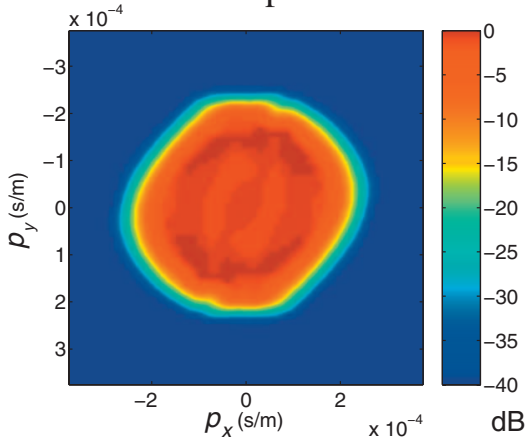

c)

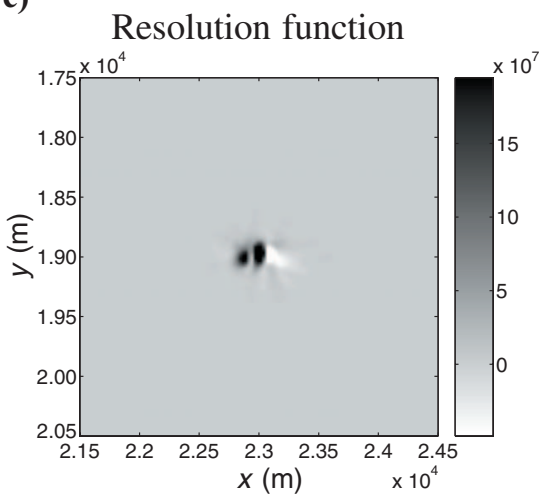

AVP imprint

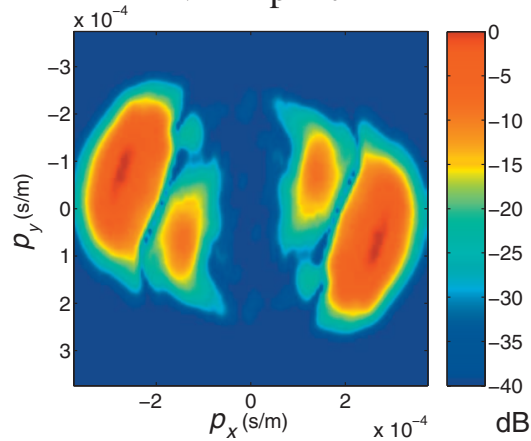

Figure 12. Resolution functions and AVP imprints for three subsalt target points: (a) at $(x, y, z)=(21.2,19,3.3) \mathrm{km},(\mathrm{b})$ at $(x, y, z)$ $=(25,31,6) \mathrm{km}$, and $(\mathrm{c})$ at $(x, y, z)=(23,19,6) \mathrm{km}$, for a full 3D geometry. The acquisition area is fully covered with closely spaced sources and detectors. 
a)

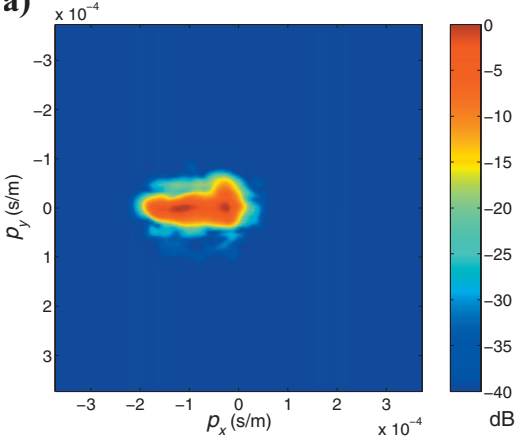

b)

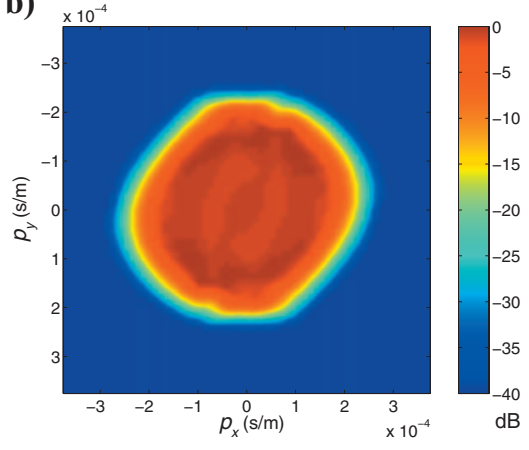

Figure 13. Estimated AVP imprints at a subsalt target point in the Ziggy model located at $(x, y, z)=(25,31,6) \mathrm{km}$, (a) for a narrow streamer geometry and (b) a full 3D geometry. The acquisition geometry corresponding to the AVP imprint shown in (a) is given by Figure 14 . The conventional streamer geometry strongly limits the range of illumination angles.

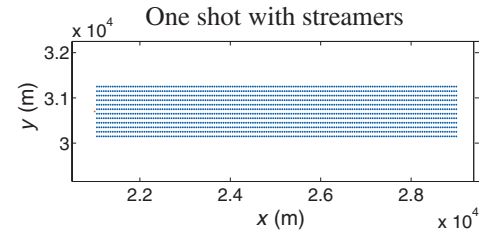

- Single source, source spacing $100 \mathrm{~m}$

- 12 streamers, cable length $8000 \mathrm{~m}$

- Cable separation of $100 \mathrm{~m}$

- Hydrophone spacing of $50 \mathrm{~m}$

- 10 sail lines

- 600-m sail line spacing

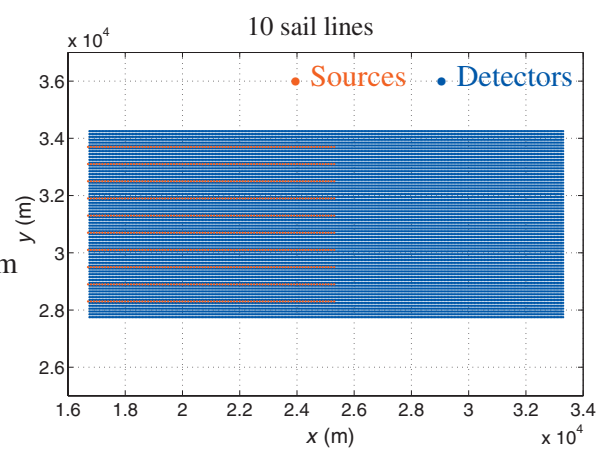

Figure 14. Streamer geometry for the results in Figure 13a. On the left, one source with streamers. On the right, all sources and streamers.

- Single source, source spacing $50 \mathrm{~m}$

- 10 streamers, cable length $9000 \mathrm{~m}$

- Cable separation $100 \mathrm{~m}$

- Hydrophone spacing $50 \mathrm{~m}$

- 1 sail line

- Sources

- Detectors
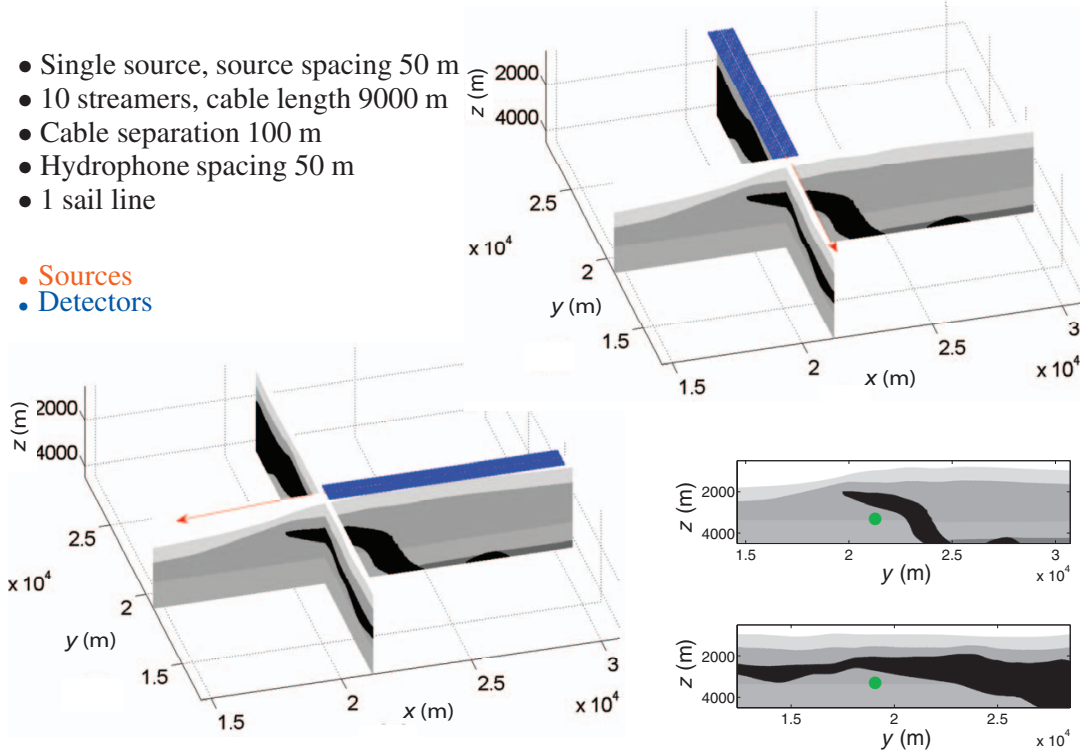

Figure 15. Two single sail lines used to generate the results given in Figure 16b. The bottom right images show two slices of the macro model in which the target point, located at $(x, y, z)=(21.2,19,3.3) \mathrm{km}$, is indicated by a green dot. result from element-by-element multiplication of these focal beams with their flipped versions (equation B-13) that are obtained by reversing the $p_{x}$-axis and $p_{y}$-axis in practice.

The resolution functions (lateral resolution at target depth) in Figure 12a and c show slight asymmetric effects because of asymmetric illumination. The resolution function in Figure $12 \mathrm{~b}$ shows a somewhat wider peak, or dot, caused by the smaller spatial bandwidth.

The AVP imprints show distinct differences. The AVP imprint in Figure 12b is the only one of these three showing a uniform range of amplitudes for all azimuths, for angles from normal incidence up to approximately $40^{\circ}$.

The AVP imprints in Figure 12a and c are clearly azimuth-dependent. Furthermore, the AVP imprints illustrate gaps that are obviously related to the illumination gaps observed in the corresponding focal beams. For the target point at $(x, y, z)$ $=(23,19,6) \mathrm{km}$, amplitudes for normal incidence and for small incidence angles are $40 \mathrm{~dB}$ or more below the maximum amplitude. Note that these are AVP imprints for full 3D acquisition geometries. These acquisition geometries can be improved only by extending the acquisition aperture. Clearly, this will not fill the gaps.

\section{Streamer acquisition}

A full 3D acquisition geometry is not feasible in practice, especially in a marine situation. Figure 13 illustrates the difference between the AVP imprint for a full 3D acquisition geometry and a conventional marine geometry. The marine geometry, displayed in Figure 14, has 10 sail lines with 600-m sail-line spacing, 12 cables per shot (with a cable length of $8000 \mathrm{~m}$ and cable spacing of $100 \mathrm{~m}$ ), 50-m inline hydrophone spacing, and $100-\mathrm{m}$ inline (single) source spacing. Figure 13a shows that the streamer geometry covers a very limited range of angles and azimuths, as is typical for conventional marine geometries.

\section{Narrow and dual-azimuth acquisition}

For the target point at $(x, y, z)=(21.2$, 19,3.3) $\mathrm{km}$, we have seen the image quality for a full $3 \mathrm{D}$ acquisition geometry (Figure 12a). Now we will see what quality remains if single sail lines are used instead of the full 3D acquisition geometry. Three cases are considered: a single sail line parallel to the $x$-axis, a single sail line parallel to the $y$-axis, and the two sail lines combined. The latter case represents the above-mentioned multiazimuth acquisition (Page et al., 2007; Keggin et al., 2006), although only two acquisition azimuths are involved. The two orthogonal sail lines are shown in Figure 15.

Resolution functions for these single sail lines are not representative of the resolution obtained 
in a streamer survey with more sail lines. However, the AVP imprints are representative of a complete streamer survey because more sail lines will not add much specular reflection energy for this target point. Of course, when more target points are considered, the analy- sis for single sail lines is not representative of the results for a streamer survey with more sail lines.

We illustrate the results in Figure 16. For comparison, Figure 16a depicts results for a one-dimensional subsurface, whereas Figure a)
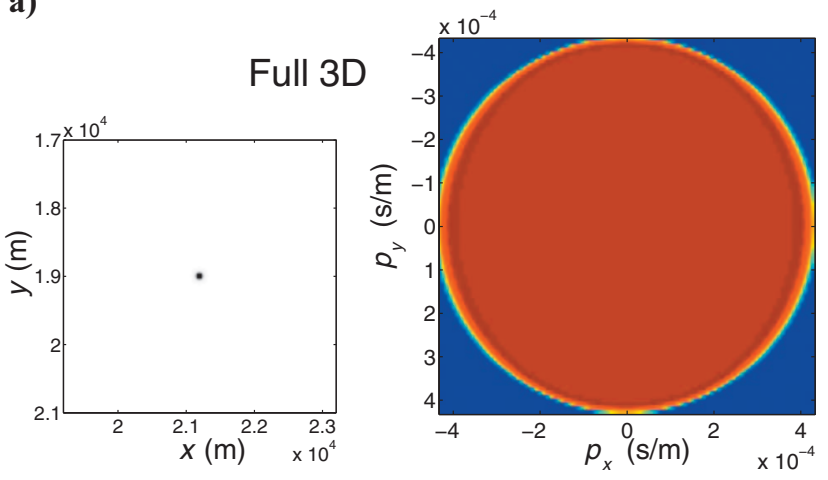

Sail line $x$-direction
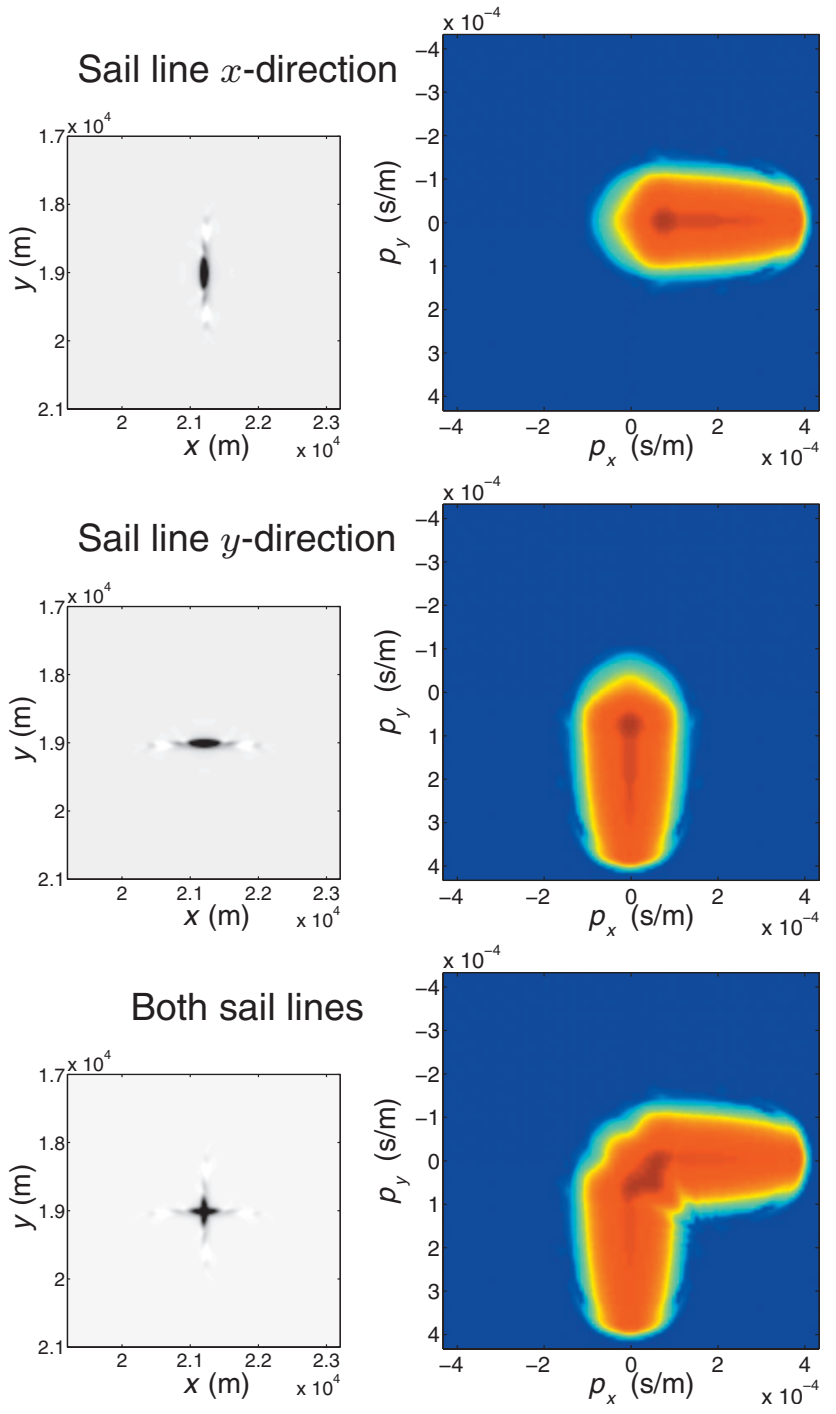

b)
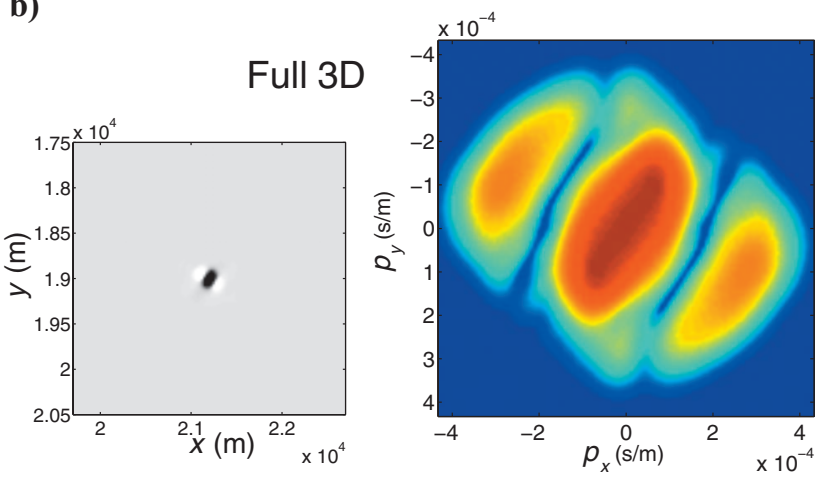

Sail line $x$-direction
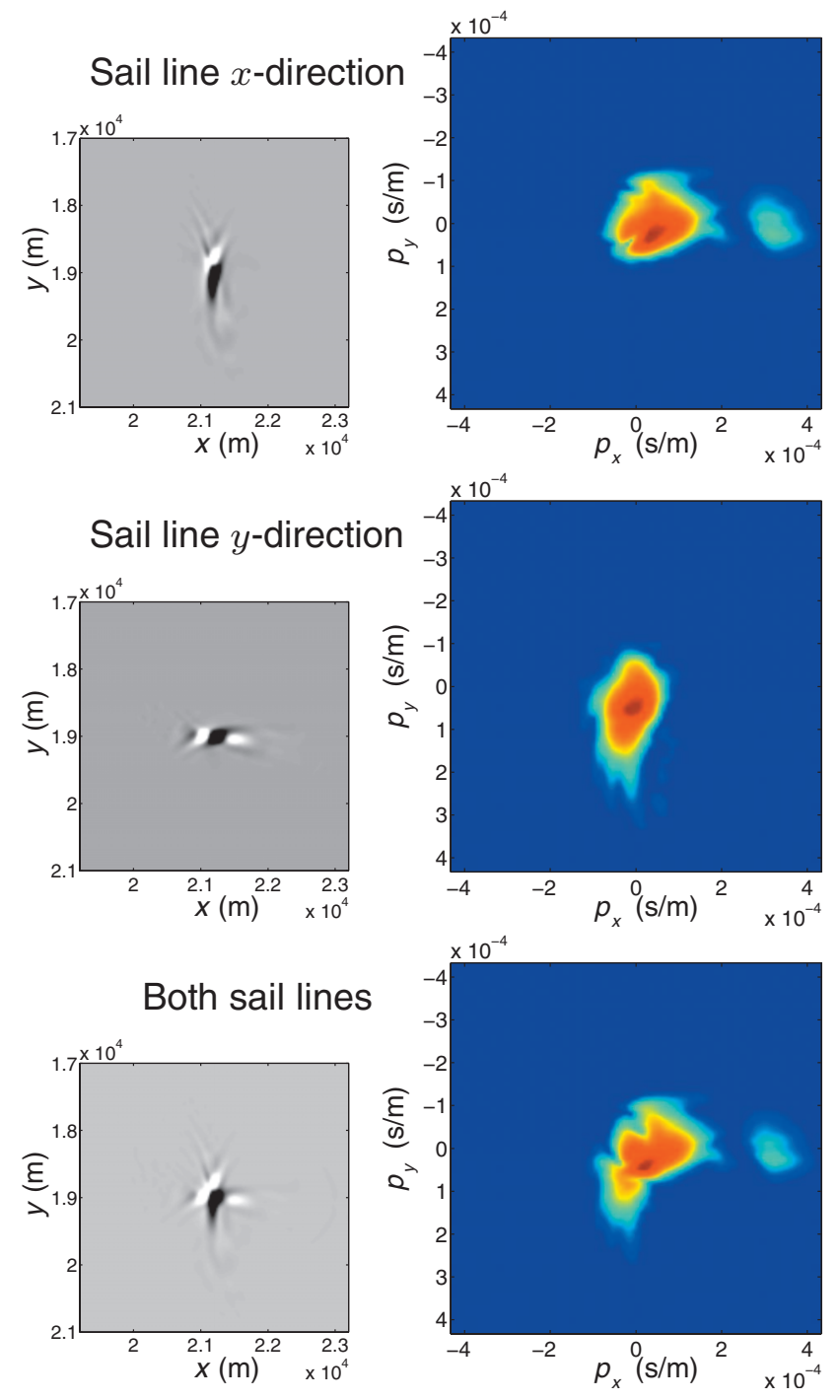

Figure 16. Resolution functions and AVP imprints (a) for a target point located at $(x, y, z)=(21.2,19,3.3) \mathrm{km}$ in a one-dimensional subsurface, and (b) in the Ziggy model, for a full 3D acquisition geometry, for one sail line in the $x$-direction, for one sail line in the $y$-direction, and for both sail lines combined. The resolution functions are the small gray-scale images. AVPimprints are the larger images, in which the color scale ranges from $-40 \mathrm{~dB}$ (blue) to $0 \mathrm{~dB}$ (red). 
$16 \mathrm{~b}$ illustrates the results for the Ziggy model. The one-dimensionalmodel has the same sediments as the Ziggy model, but interfaces are flat and the salt body is absent. Velocity ranges from $1500 \mathrm{~m} / \mathrm{s}$ at the surface to $2332 \mathrm{~m} / \mathrm{s}$ at target depth. Results for the 1D model are obtained by phase-shift extrapolation in the wavenumber-frequency domain, for a propagation angle up to $90^{\circ}$. A frequency range of 4 to $30 \mathrm{~Hz}$ is used for both the 1D model and the Ziggy model.

For the 1D situation, the result is very straightforward. For the full $3 \mathrm{D}$ geometry, there is no AVP imprint — the amplitudes are uniform for all angles and azimuths. AVP imprints for the two sail lines clearly show the expected acquisition-related azimuth limitation. As the AVP imprint for the full 3D geometry is azimuth-independent, the two sail lines yield the same angle-dependent amplitude information, assuming that the target reflectivity is azimuth-independent. Combining the two sail lines into dual-azimuth acquisition shapes the resolution function and yields an improved AVP imprint that includes the contributions of both sail lines. This means that amplitude information is now preserved for both azimuths. This can be of high added value if the target reflectivity is azimuth-dependent.

For the Ziggy model, the AVP imprint for the full 3D geometry is not azimuth-independent. This is a natural consequence of 3D structural complexities in the model. As discussed previously, gaps appearing in the AVP imprint for this target point result from postcritical wave incidence at the base of salt - a structure-related limitation. As a consequence, both structural and acquisition-related limitations play a part for the two single sail lines. This yields a severe limitation of the AVP information.

For this complex model, the acquisition geometry at the surface does not translate directly into angles at the target. Still, it can be noticed that the full 3D AVP imprint gives a good indication of what limitations from the subsurface structure can be expected for the two sail directions.

As with the 1D model, the AVP imprint for the Ziggy model (slightly) extends when the two sail lines are combined. As a next

Table 1. Medium properties used for forward modeling of $P$-P reflectivity. Layer $i$ refers to the layer above the target point, and layer $i+1$ refers to the layer below the target point.

\begin{tabular}{lccc} 
& $V_{\mathrm{P}}(\mathrm{m} / \mathrm{s})$ & $V_{\mathrm{S}}(\mathrm{m} / \mathrm{s})$ & $\rho\left(\mathrm{kg} / \mathrm{m}^{3}\right)$ \\
\hline Layer $i$ & 2700 & 1400 & 1800 \\
Layer $i+1$ & 2800 & 1500 & 1900 \\
\hline
\end{tabular}

Table 2. Estimated property contrasts. Estimation a refers to the full coverage geometry, estimation b refers to the marine geometry. The notation $\delta \ln \rho$ stands for $\ln \rho_{i+1} / \rho_{i}\left(\operatorname{similarly}\right.$ for $\delta \ln V_{\mathrm{P}} \rho$ and $\left.\delta \ln V_{\mathrm{S}} \rho\right)$.

\begin{tabular}{lcccccc}
\hline & $\delta \ln V_{\mathrm{P}} \rho$ & $\delta \ln V_{\mathrm{S}} \rho$ & $\delta \ln \rho$ & $\begin{array}{c}V_{\mathrm{P}, i+1} \\
(\mathrm{~m} / \mathrm{s})\end{array}$ & $\begin{array}{c}V_{\mathrm{S}, i+1} \\
(\mathrm{~m} / \mathrm{s})\end{array}$ & $\begin{array}{c}\rho_{i+1} \\
\left(\mathrm{~kg} / \mathrm{m}^{3}\right)\end{array}$ \\
\hline Actual & 0.0890 & 0.123 & 0.0541 & 2800 & 1500 & 1900 \\
Estimation a & 0.0792 & 0.118 & 0.106 & 2633 & 1417 & 2001 \\
Estimation b & 0.0331 & 0.0125 & 0.391 & 1891 & 959 & 2660 \\
\hline
\end{tabular}

step, it can be considered to use orthogonal sail lines simultaneously. In the target area, this will yield more amplitude information for a larger range of azimuths.

\section{Impact on inversion}

Computed AVP imprints can be used to investigate the impact of the acquisition geometry on amplitude inversion. Using a forward model for reflectivity, undistorted reflection amplitudes as a function of ray parameter are computed for a known (chosen) set of contrast parameters at the interface where the target point is located. The AVP imprint is superposed on the undistorted reflection function. (Note that the undistorted reflectivity curve with superposed AVP imprint represents the AVA amplitude variation with angle information that usually would be extracted from the seismic data.) The imprinted reflection amplitudes are inverted to find a set of estimated contrast parameters. The difference between known and estimated contrasts shows the (isolated) impact of acquisition-related amplitude distortions of the signal. Of course, other distortions caused by, e.g., coherent noise, incoherent noise, and wavelet stretch are not taken into account. For a fair impression of the total distortion of the inversion, these effects should be incorporated in the analysis.

We apply this procedure to the AVP imprints in Figure 13. We use an azimuth-independent forward-reflectivity model valid for a large range of precritical angles (Dey and Gisolf, 2007). Subsurface properties (P-wave velocity $V_{\mathrm{P}}$, S-wave velocity $V_{\mathrm{S}}$, density $\rho$ ) are specified in Table 1. These properties correspond to a target interface in the Ziggy model.

The results of the inversion are given in Table 2, where actual and estimated medium property contrasts are listed. To indicate the consequences of these inversion results, Table 2 also gives medium properties for layer $i+1$ (if the properties of layer $i$ are known). This illustrates that errors in the estimated medium properties are up to $6 \%$ for the full 3D geometry, whereas errors are between $30 \%$ and $40 \%$ for the narrow-azimuth geometry.

Figures $17 \mathrm{a}$ and $\mathrm{b}$ show the $\mathrm{P}-\mathrm{P}$ reflectivity curves, as a function of ray parameter $p$ : black lines represent undistorted reflectivity, blue lines represent undistorted reflectivity with amplitude imprint, and red lines depict reflectivity curves as estimated by inversion of the imprinted amplitudes. This figure reconfirms that the narrow streamer geometry has a devastating effect on angle-dependent amplitudes. As a next step, the estimated reflectivity curves could serve as a basis for a ray-parameter-dependent amplitude correction.

\section{DISCUSSION}

In the previous section, we discussed a modelbased acquisition geometry analysis by focal beams. The method is suitable as part of our envisaged acquisition design strategy, which consists of the following elements:

- The best attainable image quality for specific wave type(s) is given by assessment of a full 3D wide-azimuth, well-sampled, stationary acquisition geometry. For complex subsurfaces, the result reveals the fundamental limitations of the involved wave type(s). 
- The geometry-related image artifacts and (angle-dependent) amplitude imprints can be determined by analyzing the focal source and focal detector beams for the sparse source and detector geometries that we see in practice.

- Analysis results indicate the options for improvement of image quality and characterization accuracy.

- Choice of wave type(s).

- Adjustment of the acquisition system. Adjustments can be made to the source and/or detector geometry.

- Corrections to the data. For weak distortions, angle-dependent amplitude corrections can be applied by postprocessing.

Note that the rising trend of recording more azimuths (wide-, multi-, and rich-azimuth acquisition) addresses the above-mentioned strong limitation of azimuths that is observed for conventional towed-streamer geometries. In these new acquisition designs, a larger range of azimuths is covered. Thus, the full $3 \mathrm{D}$ acquisition is more closely approximated, resulting in better illumination and better multiple suppression (Moldoveanu and Egan, 2006; Corcoran et al., 2007; Page et al., 2007; and works referenced by these authors).

However, in the example we have seen that a complex overburden with high velocity contrasts yields severe AVP imprints even for a full 3D acquisition geometry. This indicates that certain defects in image quality cannot be mended easily by a more sophisticated migration or by changes to the (surface) acquisition geometry. Instead, image quality improvement could be achieved by:

- The use of lower frequencies, which improve the illumination and detection of the target. Using low frequencies for imaging beneath high-velocity structures has been addressed previously by Ziolkowski et al. (2003) and Kapoor et al. (2005).

- The use of different wave type combinations that minimize velocity contrasts, like P-S-P waves, as discussed by Alá'i and Verschuur (2006).

- Application of fundamentally different seismic acquisition setups. Examples are the use of vertical seismic profiles (VSPs), see, e.g., Campbell et al. (2006), and virtual-source seismic monitoring in horizontal and deviated wells (Bakulin et al., 2007).
- The use of multiple reflections for imaging. Instead of considering the multiples as noise, they can be considered as signal that potentially illuminates different areas in the subsurface (Jiang et al., 2005).

Note that the velocity model in our method is the macro velocity model, similar to what is used in migration. If large uncertainties exist about the macro velocity model, different scenarios should be used and the sensitivity on the optimum acquisition geometry should be evaluated. The more uncertainty about the subsurface, the less specific the acquisition geometry should be.

The method provides further analysis opportunities such as P-S acquisition ( $\mathrm{P}$ focal beam combined with $\mathrm{S}$ focal beam) and acquisition with buried detectors (VSP). Current research includes focal beam analysis on coherent noise (Al-Ali, 2007).

\section{CONCLUSIONS}

Our method provides insight into illumination and sensing problems in complex 3D media. Because wave-equation modeling is used, frequency-dependent effects can be investigated. Low frequencies are less sensitive to irregular salt structures than higher frequencies. The chosen implementation uses recursive depth extrapolation starting at the target depth. Therefore, the most easily computed situation is the all-azimuth full 3D acquisition, representing the best migration result that could be obtained in theory: it shows fundamental image limitations caused by the complex subsurface and by the migration method (one-way wave-equation in this case). This provides a straightforward way to identify whether it is possible to image certain problematic subsalt locations from the surface. Results for the Ziggy model reveal gaps in angle and azimuth ranges of illumination (or sensing). The main cause is scattering at the salt boundaries. Dip limitation in the migration operators is the second main cause. Subsalt AVP imprints depend strongly on location with respect to the salt body. Beneath a dipping flank of the salt, postcritical wave incidence will occur even for low angles. The AVP imprint indicates to what extent amplitude inversion can be hampered by acquisition-related amplitude inaccuracies. As expected, the distorting effect of a conventional, narrow, marine geometry is significant. Because traditional marine streamer acquisition limits the angle and azimuth range strongly, illumination gaps should be considered when choosing the azimuth(s) of the sail lines. Wide-, multi-, and rich-azimuth marine acquisition techniques can provide a closer approximation of the full 3D acquisition

\section{ACKNOWLEDGMENTS}

The authors thank sponsors of the DELPHI consortium for their support. The first author is grateful to Jan Thorbecke for use of his wave extrapolation software. We acknowledge assistant editor Vladimir Grechka, the associate editor, reviewers Vetle Vinje and Peter Pecholcs, and one anonymous reviewer for their constructive com-
Figure 17. (a) and (b) show the undistorted reflectivity (black), reflectivity with amplitude imprint (blue), and estimated reflectivity (red), as a function of ray parameter $p$, for the narrow streamer geometry and the full 3D (all-azimuth) geometry, respectively. geometry.

ments that helped us improve this paper.

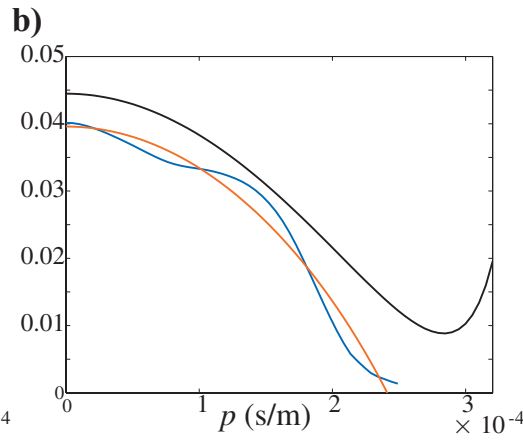




\section{APPENDIX A \\ DIFFERENT FORMULATIONS OF FOCAL BEAMS}

According to equations 6 and 8 in the theory section, the focal beams at depth level $z_{m}, \mathbf{D}_{j}^{\dagger}\left(z_{m}, z_{m}\right)$ and $\mathbf{S}_{j}\left(z_{m}, z_{m}\right)$, are defined as

$$
\mathbf{D}_{j}^{\dagger}\left(z_{m}, z_{m}\right)=\mathbf{F}_{j}^{\dagger}\left(z_{m}, z_{0}\right) \mathbf{D}\left(z_{0}\right) \mathbf{W}\left(z_{0}, z_{m}\right),
$$

and

$$
\mathbf{S}_{j}\left(z_{m}, z_{m}\right)=\mathbf{W}\left(z_{m}, z_{0}\right) \mathbf{S}\left(z_{0}\right) \mathbf{F}_{j}\left(z_{0}, z_{m}\right),
$$

respectively. Alternative definitions of the focal source beam and focal detector beam at depth level $z_{m}$ are (Volker, 2002):

$$
\begin{aligned}
& \mathbf{D}_{j}\left(z_{m}, z_{m}\right)=\mathbf{F}\left(z_{m}, z_{0}\right) \mathbf{D}\left(z_{0}\right) \mathbf{W}_{j}\left(z_{0}, z_{m}\right), \\
& \mathbf{S}_{j}^{\dagger}\left(z_{m}, z_{m}\right)=\mathbf{W}_{j}^{\dagger}\left(z_{m}, z_{0}\right) \mathbf{S}\left(z_{0}\right) \mathbf{F}\left(z_{0}, z_{m}\right) .
\end{aligned}
$$

Depending on the choice of wavefield propagator, using these definitions of the focal beams to compose the resolution function and AVP imprint (see Appendix B) can be more convenient from a computational point of view.

If the inverse wavefield extrapolator $\mathbf{F}$ is approximated by its matched filter and transmission effects are neglected, the alternative definitions are the complex conjugate transposed versions of the original definitions:

$$
\begin{aligned}
& \mathbf{S}_{j}^{\dagger}\left(z_{m}, z_{m}\right)=\left[\mathbf{S}_{j}\left(z_{m}, z_{m}\right)\right]^{H}, \\
& \mathbf{D}_{j}\left(z_{m}, z_{m}\right)=\left[\mathbf{D}_{j}^{\dagger}\left(z_{m}, z_{m}\right)\right]^{H} .
\end{aligned}
$$

Superscript $H$ indicates complex conjugate transposition. Expressions A-5 and A- 6 tell that in the space-time domain, $\mathbf{D}_{j}\left(z_{m}, z_{m}\right)$ is the time-reversed version of $\mathbf{D}_{j}^{\dagger}\left(z_{m}, z_{m}\right)$ and $\mathbf{S}_{j}^{\dagger}\left(z_{m}, z_{m}\right)$ is the time-reversed version of $\mathbf{S}_{j}\left(z_{m}, z_{m}\right)$.

\section{APPENDIX B}

\section{RESOLUTION FUNCTION AND AVP IMPRINT}

The resolution function is the confocal imaging result of the response of grid-point $j$ at depth $z_{m}$ :

$$
\delta_{j} \mathbf{P}_{i h}\left(z_{m}, z_{m}\right)=\mathbf{F}_{i}^{\dagger}\left(z_{m}, z_{0}\right) \delta_{j} \mathbf{P}\left(z_{0}, z_{0}\right) \mathbf{F}_{h}\left(z_{0}, z_{m}\right),
$$

with $i=h$ varying around focal point $j$. These indices all refer to lateral coordinate pairs at the target depth level $z_{m}$.

Confocal means that the two focusing operators are applied for the same subsurface grid point $(i=h) . \delta_{j} \mathbf{P}\left(z_{0}, z_{0}\right)$ is the angle-independent grid-point response:

$$
\delta_{j} \mathbf{P}\left(z_{0}, z_{0}\right)=\mathbf{D}\left(z_{0}\right) \mathbf{W}\left(z_{0}, z_{m}\right) \delta_{j} \mathbf{R}\left(z_{m}, z_{m}\right) \mathbf{W}\left(z_{m}, z_{0}\right) \mathbf{S}\left(z_{0}\right) .
$$

$\delta_{j} \mathbf{R}\left(z_{m}, z_{m}\right)$ represents the angle-independent grid-point reflectivity matrix at lateral location $j$ :

$$
\begin{aligned}
& \delta_{j} \mathbf{R}_{h i}=1 \quad i=h=j, \\
& \delta_{j} \mathbf{R}_{h i}=0 \quad i \neq j \quad \text { or } h \neq j .
\end{aligned}
$$

Each value of $i(=h)$ in equation B-1 gives one element of the total resolution function. Considering the formulation of the focal beams as equations 6 and 8, it appears that each element of the resolution function is obtained by using a different pair of a focal detector beam and a focal source beam. In practice, computation of the resolution function can be simplified by using the time-reversed versions of the focal beams, equations A-3 and A-4. In that case, the resolution function can be obtained by an element-by-element multiplication of one focal source beam and one focal detector beam. This can be seen by substituting equation B-2 and the reflectivity matrix $\delta_{j} \mathbf{R}\left(z_{m}, z_{m}\right)$ (expressions B-3) in equation B-1:

$$
\begin{aligned}
\delta_{j} \mathbf{P}_{i h}\left(z_{m}, z_{m}\right)= & \mathbf{F}_{i}^{\dagger}\left(z_{m}, z_{0}\right) \mathbf{D}\left(z_{0}\right) \mathbf{W}\left(z_{0}, z_{m}\right) \delta_{j} \mathbf{R}\left(z_{m}, z_{m}\right) \\
& \times \mathbf{W}\left(z_{m}, z_{0}\right) \mathbf{S}\left(z_{0}\right) \mathbf{F}_{h}\left(z_{0}, z_{m}\right) \\
= & \mathbf{F}_{i}^{\dagger}\left(z_{m}, z_{0}\right) \mathbf{D}\left(z_{0}\right) \mathbf{W}_{j}\left(z_{0}, z_{m}\right) \\
& \times \mathbf{W}_{j}^{\dagger}\left(z_{m}, z_{0}\right) \mathbf{S}\left(z_{0}\right) \mathbf{F}_{h}\left(z_{0}, z_{m}\right) \\
= & \mathbf{D}_{i j}\left(z_{m}, z_{m}\right) \mathbf{S}_{j h}\left(z_{m}, z_{m}\right),
\end{aligned}
$$

for $h=i=-N \ldots N$, where $2 N+1$ is the number of points in the target area that is included in the focal beams. In this expression, $\mathbf{D}_{i j}\left(z_{m}, z_{m}\right)$ and $\mathbf{S}_{j h}\left(z_{m}, z_{m}\right)$ are single elements that correspond to elements of the focal beams of equations A-3 and A-4, respectively. Therefore the resolution function is an element-by-element multiplication of the focal detector beam and the focal source beam in the space-frequency domain.

The angle-dependent amplitude imprint for a plane reflecting boundary (an area around the considered grid point), is the bifocal imaging result for an angle-independent reflector:

$$
\Delta \mathbf{P}_{i j}\left(z_{m}, z_{m}\right)=\mathbf{F}_{i}^{\dagger}\left(z_{m}, z_{0}\right) \Delta \mathbf{P}\left(z_{0}, z_{0}\right) \mathbf{F}_{j}\left(z_{0}, z_{m}\right),
$$

in which $i$ and $j$ indicate lateral coordinate pairs $(x, y)_{i}$ and $(x, y)_{j}$ at the target depth level $z_{m}$. The term bifocal means that the two focusing operators are applied for subsurface grid points with different lateral locations $(i \neq h) . \Delta \mathbf{P}\left(z_{0}, z_{0}\right)$ is the seismic response of a reflector with angle-independent (and unit) reflectivity — in that case, the reflectivity matrix $\mathbf{R}$ is an identity matrix:

$$
\Delta \mathbf{P}\left(z_{0}, z_{0}\right)=\mathbf{D}\left(z_{0}\right) \mathbf{W}\left(z_{0}, z_{m}\right) \mathbf{I}\left(z_{m}\right) \mathbf{W}\left(z_{m}, z_{0}\right) \mathbf{S}\left(z_{0}\right) .
$$

For a range of grid-points $i$, the bifocal imaging result is

$$
\Delta \mathbf{P}_{j}\left(z_{m}, z_{m}\right)=\mathbf{F}\left(z_{m}, z_{0}\right) \Delta \mathbf{P}\left(z_{0}, z_{0}\right) \mathbf{F}_{j}\left(z_{0}, z_{m}\right),
$$

in which each row of $\mathbf{F}\left(z_{m}, z_{0}\right)$ contains a focusing operator for one grid-point $i$. The bifocal imaging expression can be written as the product of a focal source beam and a focal detector beam (both are vectors) by combining equations B-5 and B- 6 , and equations 6 and 8 . For one grid-point $i$, this yields

$$
\Delta \mathbf{P}_{i j}\left(z_{m}, z_{m}\right)=\mathbf{D}_{i}^{\dagger}\left(z_{m}, z_{m}\right) \mathbf{S}_{j}\left(z_{m}, z_{m}\right),
$$

and for a range of grid-points $i$,

$$
\Delta \mathbf{P}_{j}\left(z_{m}, z_{m}\right)=\mathbf{D}\left(z_{m}, z_{m}\right) \mathbf{S}_{j}\left(z_{m}, z_{m}\right),
$$

in which every row of $\mathbf{D}\left(z_{m}, z_{m}\right)$ contains the focal beam for a gridpoint $i$. The AVP imprint for grid-point $j$ can be obtained from the bifocal imaging results for a range of grid-points $i$. Therefore, it appears that the AVP imprint is obtained by using one focal source 
beam for grid-point $j$, and many focal detector beams for all gridpoints $i$. In practice, computation of the AVP imprint is simplified by assuming that the focal detector beam does not vary as a function of location $i$ around the target point $j$. For each point $i$ in that case, a shifted version of focal detector beam $\mathbf{D}_{j}^{\dagger}\left(z_{m}, z_{m}\right)$ can be used, and the bifocal imaging result can be written as

$$
\begin{aligned}
\Delta \mathbf{P}_{j}\left(z_{m}, z_{m}\right) & =\mathbf{D}\left(z_{m}, z_{m}\right) \mathbf{S}_{j}\left(z_{m}, z_{m}\right)=\mathbf{D}^{H}\left(z_{m}, z_{m}\right) \mathbf{S}_{j}\left(z_{m}, z_{m}\right) \\
& =\left[\mathbf{D}^{T}\right]^{*}\left(z_{m}, z_{m}\right) \mathbf{S}_{j}\left(z_{m}, z_{m}\right) .
\end{aligned}
$$

This equation reveals that each element of $\Delta \mathbf{P}_{j}\left(z_{m}, z_{m}\right)$ is obtained by a space-invariant convolution:

$$
\Delta \mathbf{P}_{i j}\left(z_{m}, z_{m}\right)=\sum_{v=-N}^{N} \mathbf{D}_{j(i-v)}^{*}\left(z_{m}, z_{m}\right) \mathbf{S}_{v j}\left(z_{m}, z_{m}\right),
$$

which can be written in its functional form as

$$
\Delta P_{j}\left(\mathbf{x}_{i}, z_{m}\right)=\sum_{v=-N}^{N} D_{j}^{*}\left(\mathbf{x}_{i}-\mathbf{x}_{v}, z_{m}, \omega\right) S_{j}\left(\mathbf{x}_{v}, z_{m}, \omega\right),
$$

in which $\mathbf{x}=(x, y)$. This convolution is a multiplication in the Radon domain. Furthermore, complex conjugation in the space-frequency domain implies reversal of the ray parameter in the Radon domain. Consequently, the AVPimprint is

$$
\Delta \tilde{P}_{j}\left(\mathbf{p}, z_{m}, z_{m}\right)=\tilde{D}_{j}\left(-\mathbf{p}, z_{m}\right) \tilde{S}_{j}\left(\mathbf{p}, z_{m}\right)
$$

in which $\mathbf{p}=\left(p_{x}, p_{y}\right)$. In short, the AVP imprint can be approximated by an element-by-element multiplication of the Radon-transformed focal detector beam and focal source beam with focal point location $j$.

\section{REFERENCES}

Al-Ali, M. N., 2007, Land seismic data acquisition and preprocessing: Ph.D. thesis, Delft University of Technology.

Alá'i, R., and D. J. Verschuur, 2006, Subsalt imaging of wave converted reflections in physical model data: 68th Annual Conference and Technical Exposition, EAGE, Extended Abstracts, P268.

Bakulin, A. V., J. L. Lopez, I. Sinha Herhold, and A. A. Mateeva, 2007, Onshore monitoring with virtual-source seismic in horizontal wells: Challenges and solutions: 77th Annual International Meeting, SEG, Expanded Abstracts, 2893-2897.

Berkhout, A. J., 1982, Seismic migration, imaging of acoustic energy by wavefield extrapolation, A. Theoretical aspects: Elsevier.

_ 1997, Pushing the limits of seismic imaging, Part I: Prestack migration in terms of double dynamic focusing: Geophysics, 62, 937-953.

_ 2004, The data-driven seismic value chain, providing a business context for the velocity issue: Geophysical Prospecting, 52, 481-487.

Berkhout, A. J., L. Ongkiehong, A. W. F. Volker, and G. Blacquière, 2001, Comprehensive assessment of seismic acquisition geometries by focal beams - Part I: Theoretical considerations: Geophysics, 66, 911-917.

Beylkin, G., 1985, Imaging of discontinuities in the inverse scattering problem by inversion of a causal generalized Radon transform: Journal of Mathematical Physics, 26, 99-108.

Blacquière, G., H. W. J. Debeye, C. P. A. Wapenaar, and A. J. Berkhout, 1989 3D table-driven migration: Geophysical Prospecting, 37, 925-958.

Brink, M., N. Jones, J. Doherty, V. Vinje, and R. Laurain, 2004, Infill decisions based on estimated reflection amplitudes: 74th Annual International Meeting, SEG, Expanded Abstracts, 57-60.
Campbell, A., L. Nutt, R. Smith, and H. Chang, 2006, Designing borehole seismic VSPs for complex subsalt or near-salt reservoir evaluation: First Break, 24, 69-74.

Campbell, S., W. B. Pramik, and B. Cafarelli, 2002, Comparative ray-based illumination analysis: 72nd Annual International Meeting, SEG, Expanded Abstracts, 41-44.

Chang, T., C. Kue, L. Canales, and C. Shih, 2002, Forward modeling attribute analysis for AVO and prestack depth migration: 72nd Annual International Meeting, SEG, Expanded Abstracts, 48-51.

Corcoran, C., C. Perkins, C. D. Lee, P. A. Cattermole, R. Cook, and N. Moldoveanu, 2007, A wide-azimuth streamer acquisition pilot project in the Gulf of Mexico: The Leading Edge, 26, no. 4, 460-468.

de Bruin, C. G. M., C. P. A. Wapenaar, and A. J. Berkhout, 1990, Angle-dependent reflectivity by means of prestack migration: Geophysics, $\mathbf{5 5}$, 1223-1234.

Dey, A. K., and A. Gisolf, 2007, Wide-angle linear forward modeling of synthetic seismograms: Geophysical Prospecting, 55, no. 5, 707-718.

Dong, L., X. Cai, P. Li, K. Yang, and S. Liu, 2005, Seismic wave illumination for Yumen overthrust structure and seismic survey optimal design: 75th Annual International Meeting, SEG, Expanded Abstracts, 48-51.

Gibson, R. L., and C. Tzimeas, 2002, Quantitative measures of image resolution for seismic survey design: Geophysics, 67, 1844-1852.

Holberg, O., 1988, Towards optimum one-way wave propagation: Geophysical Prospecting, 36, 99-114

Jiang, Z., J. Yu, G. T. Schuster, and B. E. Hornby, 2005, Migration of multiples: The Leading Edge, 24, 315-318.

Jurick, D., J. Codd, F. Hoxha, J. Naumenko, and D. Kessler, 2003, Gulf of Suez acquisition design using 2D and 3D full wave equation simulation: 73rd Annual International Meeting, SEG, Expanded Abstracts, 2136-2139.

Kapoor, J., C. Stork, and M. S. Egan, 2005, Benefits of low frequencies for subsalt imaging: 75th Annual International Meeting, SEG, Expanded Abstracts, 1993-1996.

Keggin, J., T. Manning, W. E. A. Rietveld, C. Page, E. Fromyr, and R. van Borselen, 2006, Key aspects of multi-azimuth acquisition and processing: 76th Annual International Meeting, SEG, Expanded Abstracts, 2886-2890

Laurain, R., J. Mispel, and V. Vinje, 2002, Towards better amplitude maps by simulated migration: 72nd Annual International Meeting, SEG, Expanded Abstracts, 1376-1379.

Lecomte, I., and L. Pochon-Guerin, 2005, Simulated 2D/3D PSDM images with a fast, robust, and flexible FFT-based filtering approach: 75th Annual International Meeting, SEG, Expanded Abstracts, 1810-1813.

Moldoveanu, N., and M. S. Egan, 2006, From narrow-azimuth to wide- and rich-azimuth acquisition in the Gulf of Mexico: First Break, 24, no. 12 69-76.

Moldoveanu, N., K. S. Lee, and P. Jorgensen, 2003, Onboard 3D \& 4D subsurface target illumination: A tool to optimize streamer marine acquisition: 73rd Annual International Meetings, SEG, Expanded Abstracts, 4-7.

Muerdter, D., and D. Ratcliff, 2001a, Understanding subsalt illumination through ray-trace modeling, Part 1: Simple 2D salt models: The Leading Edge, 20, 578-595.

- 2001b, Understanding subsalt illumination through ray-trace modeling, Part 3: Salt ridges and furrows, and the impact of acquisition orientation: The Leading Edge, 20, 803-816.

Page, C., R. G. van Borselen, E. Fromyr, J. Keggin, T. Manning, and W. E. Rietveld, 2007, Experience with towed streamer multi-azimuth processing and acquisition: First Break, 25, no. 3, 41-44.

Regone, C. J., 2006, A modeling approach to wide-azimuth design for subsalt imaging: The Leading Edge, 25, 1467-1475.

Slawson, S. E., K. D. Grove, and G. W. Fischer, 1994, Model-based 3D seismic acquisition design: 64th Annual International Meeting, SEG, Expanded Abstracts, 919-920.

Thorbecke, J. W., C. P. A. Wapenaar, and G. Swinnen, 2004, Design of oneway wavefield extrapolation operators, using smooth functions in WLSQ optimization: Geophysics, 69, 1037-1045.

Toxopeus, G., S. Petersen, and K. Wapenaar, 2003, Improved geologic modeling and interpretation by simulated migrated seismics: 73rd Annual International Meeting, SEG, Expanded Abstracts, 1829-1832.

van Veldhuizen, E. J., 2006, Integrated approach to 3D seismic acquisition geometry analysis: Ph.D. thesis, Delft University of Technology.

Vermeer, G. J. O., 1999, Factors affecting spatial resolution: Geophysics, 64, 942-953.

Vidale, J. E., 1988, Finite-difference calculation of travel times: Bulletin of the Seismological Society of America, 78, 2062-2076. 
Virieux, J., 1986, $P-S V$ wave propagation in heterogeneous media: velocitystress finite-difference method: Geophysics, 51, 889-901.

Volker, A. W. F., 2002, Assessment of 3D seismic acquisition geometries by focal beam analysis: Ph.D. thesis, Delft University of Technology.

Volker, A. W. F., G. Blacquière, A. J. Berkhout, and L. Ongkiehong, 2001, Comprehensive assessment of seismic acquisition geometries by focal beams - Part II: Practical aspects and examples: Geophysics, 66 , 918-931.
Xie, X., S. Jin, and R. Wu, 2006, Wave-equation-based seismic illumination analysis: Geophysics, 71, no. 5, S169-S177.

Ziolkowski, A., and J. T. Fokkema, 1986, Tutorial on the progressive attenuation of high-frequency energy in seismic reflection data: Geophysical Prospecting, 34, 981-1001.

Ziolkowski, A., P. Hanssen, R. Gatliff, H. Jakubowicz, A. Dobson, G. Hampson, X.-Y. Li, and E. Liu, 2003, Use of low frequencies for sub-basalt imaging: Geophysical Prospecting, 51, 169-182. 